\title{
Equity Market Response to Form 20-F Disclosures for ADR Firms
}

\author{
Michael H. Senteney ${ }^{1}$, David L. Senteney ${ }^{2} \&$ Mohammad S. Bazaz ${ }^{2}$ \\ ${ }^{1}$ Department of Economics, Ohio University, USA \\ ${ }^{2}$ Department of Accounting and Finance, California State University, San Bernardino, USA \\ Correspondence: Mohammad S. Bazaz, Department of Accounting and Finance, California State University, San \\ Bernardino, USA. Tel: 909-537-5722. E-mail: mbazaz@csusb.edu
}

Received: January 5, 2017

Accepted: February 15, 2017

Online Published: February 25, 2017

doi:10.5539/ijef.v9n3p233

URL: https://doi.org/10.5539/ijef.v9n3p233

\begin{abstract}
Non-U.S. companies may list securities in U.S. stock exchanges, provided that they file a set of audited financial statements as well as comply with extensive SEC disclosure requirements. We speculate that non-U.S. firms who choose to be listed in the major U.S. exchanges will comply with the supplemental disclosure requirements in order to have the supplemental disclosures impounded in the home country equity share price via the ADR share price in the manner described by Fishman and Hagerty (1989). We investigate the information content of non-U.S. firm's earnings released vis-à-vis the SEC Form 20-F filings in both ADR and home country equity share markets. We employed models of the ADR and equity security share earnings release date abnormal returns controlling for the incremental firm-specific SEC Form 20-F disclosures required of exchange listed ADRs. Our results suggest that both ADR and home country equity share markets exhibit abnormal returns associated with the earnings release date. Particularly noteworthy, however, is the association between magnitudes of U.S. GAAP earnings and magnitudes of SEC Form 20-F filing date. Abnormal returns are significantly larger than the association between magnitudes of reported earnings and earnings report date abnormal returns in both the ADR and home country equity share markets. Our results seemingly suggest that the U.S. ADR share market's response dominates the cross-market information flow, driving the home country equity share market response in a manner consistent with the notion that U.S. GAAP conveys price relevant information beyond reported earnings for non-U.S. firms.
\end{abstract}

Keywords: ADRs, cross listing, form 20-F, earnings announcement

\section{Introduction}

Although it may seem that firm-specific information events (e.g., earnings announcements) ought to be driven primarily by home country factors, the U.S. Securities and Exchange Commission Form 20-F disclosures for Level II and III ADRs listed on the major U.S. exchanges provide one prominent example of high-quality price-relevant disclosures arising off-shore from home- country equity markets. Extant empirical research indicates that, in numerous instances, significant information flows from the U.S. stock exchanges to the home country equity share exchanges. This suggests that U.S. stock exchanges play a preeminent role in the cross-market transmission of equity share price-relevant information (Note 1). The purpose of this study is to examine comparative aspects of the traditional earnings announcement date, abnormal returns, and the SEC Form 20-F filing date for non-U.S. firms having ADR shares traded on major U.S. stock exchanges and equity shares traded in home country equity markets.

We investigate the information content of non-U.S. firms' earnings released vis-à-vis the SEC Form 20-F filings in both ADR and home country equity share markets. We employ models of the ADR and equity share earnings release date abnormal returns while explicitly controlling for the incremental effect of the (subsequent) firm-specific SEC Form 20-F disclosures required of exchange listed ADRs. One contribution of this study is a more focused study of multiple-market information events by examining the ADR and equity share price behavior surrounding the reported earnings release, and subsequently, the U.S. Securities and Exchange Commission Form 20-F filing date. We conjecture that the SEC Form 20-F filing is a unique disclosure source providing incremental information beyond the equity share earnings releases for a number of U.S.-listed ADR firms in a manner analogous to Chen and Sami (2008) and Chen and Sami (2012) (Note 2).

The result of our empirical analyses indicates that investors in both ADR and equity share markets respond to the 
initial earnings release as well as the disclosures required by the U.S. Securities and Exchange Commission Form 20-F (which usually follow the earnings release by a number of weeks or perhaps months). For this reason, our results provide additional evidence confirming the usefulness of ADR Form 20-F information content. Particularly noteworthy, we observed that the association between the magnitudes of U.S. GAAP earnings and the magnitudes of SEC Form 20-F filing date abnormal returns is significantly larger than the association between magnitudes of reported earnings and earnings report date abnormal returns in both the ADR and home country equity share markets. The results are particularly pronounced for the difference between reported earnings and U.S. GAAP earnings. Our results seemingly suggest that the U.S. ADR share market's response dominates the cross-market information flow driving the home country equity share market's response in a manner consistent with the notion that U.S. GAAP conveys price relevant information beyond reported earnings for non-U.S. firms.

The balance of this paper is presented as follows. In the second section, we provided a brief discussion of the existing cross-market information transfer research as well as the literature relating to the U.S. Securities and Exchange Commission Form 20-F disclosures and the evidence regarding the securities market reaction to new information provided by the disclosures. The third section describes the sample selection process and describes the firms employed in the statistical analyses. The fourth section of the paper describes and discusses the empirical methods and hypotheses tests used. The fifth section presents and discusses the empirical results and accompanying robustness tests. Finally, the conclusions of this study and the suggestions for future research are presented in the final section.

\section{Extant Research Literature and Underlying Intuition}

The focus of this research regards precisely what we ought to expect regarding the role of U.S. trading in price discovery when an entity has ADR and equity shares traded simultaneously in both the United States and its home country. More specifically, are the preeminent financial disclosures required by the Securities and Exchange Commission following upon U.S. ADR share exchange listing an important determinant of whether U.S. securities exchanges play a dominant role in equity share price discovery vis-à-vis the equity shares traded on the home country exchange. The degree of correspondence between non-U.S. firms cross-listing securities on U.S stock exchanges and the informativeness of the home country equity share price is derived from the observation that higher levels of firm disclosure accompanying listings on U.S. exchanges tend to attract more investors hoping to profit from trading on the information. The numerous investors seeking to earn rents from the incremental disclosures accompanying non-U.S. firms listing shares on U.S. exchanges drives the competitive market processes which arbitrage away such profits as an integral part of price formation processes. The costly additional disclosures which the non-U.S. firms management chooses to undertake, inherent with the decision to list securities on U.S. exchanges, also tends to increase investor confidence that stock transactions occur at prices formed based upon a broad and rich set of publicly available information (Bailey et al., 2006) (Note 3).

The Securities and Exchange Commission Form 20-F filing and accompanying reconciliation to U.S. GAAP disclosures are arguably the most important source of reliable firm-specific information conveying new information beyond what is reported in accordance with home country accounting principles, in many cases, as well as at a significant cost to firm management electing preparation of the additional information required by the SEC filings. Since U.S. GAAP is generally perceived by investors as constituting the standard for high-quality accounting principles globally, the SEC Form 20-F reconciling differences with U.S. GAAP earnings and equity impose important constraints on management accounting policy choices. The constraint arises because of the need to minimize the reconciling differences with U.S. GAAP in communicating the relative success of their prospective investment projects in order for investors to perceive the ADR as maintaining high-quality reporting practices. Quite naturally, the more pronounced the differences with U.S. GAAP earnings and equity raise important question regarding earnings management practices.

We appeal to analytical research results reported in a considerable body of extant theoretical literature regarding the impact of costly voluntary management disclosures upon the equity share price formation process as the foundation of this research. Specifically, we make use of results reported by Fishman and Hagerty (1989) in which firms undertake costly voluntary disclosure and investors bear a cost of acquiring and interpreting the supplemental management disclosures (Note 4). The relevant extant research literature indicates that information environments which are supportive of market price formation processes result in equity share prices which are informative about future events. We rely largely upon Fishman and Hagerty (1989) in conjecturing that foreign firms are willing to commit to costly higher disclosure standards in order to improve the informativeness of share prices vis-a-vis future cash flows and resource allocation efficiency. U.S.-listed ADR management's commitment 
to an increased level of disclosure following upon U.S. cross-listing can have the effect of increasing the incentives for informed market participants to collect and trade on private information, and, as a result, improve U.S. listed ADR's information environment and stock price formation process. This intuition suggests that a U.S.-listed ADR's home information environment may be augmented by the additional disclosures which firm management commits to as a result of exchange required compliance with SEC regulations and U.S. GAAP. To date, however, there is limited direct evidence on the feedback relationship between a U.S. listed ADR's disclosures and the equity information environment (Note 5).

\subsection{U.S. ADR SEC Form 20-F Disclosures}

Although the Financial Reporting Policy Committee of the American Accounting Association asserts that the SEC Form 20-F Items 17 and 18 U.S. GAAP reconciliation conveys important price-relevant information to securities market investors which will impose an additional information cost upon investors when eliminated, the Financial Reporting Standards Committee of the American Accounting Association believes IFRS (i.e., without Form 20-F reconciliation) to be of sufficiently similar quality to warrant coexistence in tandem with U.S. GAAP as the single most important source of reliable (i.e., audited) company specific information available to U.S. investors at no cost via the SEC annual Form 10-K (Form 20-F) filing requirement. The Securities and Exchange Commission considers the additional opportunities for international diversification investment risk reductions provided U.S. investors as a result making listing on U.S. stock exchanges more attractive to non-U.S. firms by reducing the costs associated with SEC periodic filings (i.e., absent the Form 20-F reconciliation requirement) well worth additional information costs (i.e., if any) borne by investors as a result of discontinuing the Form 20-F reconciliation for IASB IFRS foreign private issuers (Note 6).

The extant literature provides no conclusive evidence regarding (1) the increased information costs arising from discontinuing the Form 20-F reconciliation for IASB IFRS foreign private issuers or (2) the additional international diversification benefits becoming available to U.S. investors as a result of increased numbers of Non-U.S. firms listing on U.S. stock exchanges subsequent to discontinuing the Form 20-F reconciliation requirement (Note 7). However, the SEC decision to discontinue the Form 20-F reconciliation is not uncontroversial. As mentioned previously, the American Accounting Association Financial Reporting Policy Committee (AAA 2007(a)) and Financial Reporting Standards Committee (AAA 2007(b)) arrive at different conclusions regarding interpretation of the extant research evidence and its implications for policies relating to SEC discontinuance of the Form 20-F reconciliation. Some research (e.g., Gordon et al., 2009; Henry et al., 2009; Chen \& Sami, 2008; Harris \& Muller, 1999) suggests that the Form 20-F reconciliation amounts convey price-relevant information to investors and are thereby important to investors. On the other hand, other evidence indicates that the Form 20-F reconciliation amounts are not useful to investors suggesting that IFRS quality has increased in recent years to the extent that little difference between U.S. GAAP and IFRS remains (e.g., Bartov et al., 2005; Leuz, 2003).

An extensive body of academic literature has cumulated over recent years consisting of a substantial number of research reports addressing various aspects relating to the implementation and economic/statistical properties of financial reporting employing IFRS. Soderstrom and Sun (2007) survey the extant research literature pertaining to accounting quality and IFRS implementation and point out that the greatest number of studies focus on stock price-related measures of accounting quality (e.g., value-relevance, information content, timeliness, and etcetera) concluding that these studies do not provide a comprehensive view of the usefulness of IFRS since they focus solely on how information is impounded in equity market investors' expectations. Furthermore, Bradshaw et al. (2010) find that, even though both IFRS and U.S. GAAP represent high-quality accounting standards, material reconciling items persist to the extent of establishing considerable uncertainty that IFRS constitute accounting standards which are of equivalent or higher quality when compared with U.S. GAAP.

The extant relevant research literature addressing the statistical properties of accounting financial statement amounts generated using IFRS indicates that IFRS accounting principles generate accounting measures which are of higher quality in relation to home country accounting principles with the exception of U.S. GAAP (Note 8). Barth, Landsman, and Lang (2008) use a sample of 319 IFRS reporting companies from 1990 to 2003 to provide empirical results indicating that companies using IFRS display (1) smaller degree of earning smoothing, (2) loss recognition with greater timeliness, and (3) greater value relevance than firms applying non-US domestic GAAP. Results reported by Ashbaugh and Pincus (2001) indicate that analyst forecasted errors for IFRS firms are smaller than firms using non-U.S. domestic GAAP. On the other hand, Barth, Landsman, Lang, and Williams (2006) find that IAS/IFRS firms exhibit more earning smoothing, more timely loss recognition and less pronounced relation between accounting earnings and share prices in comparing IFRS to U.S. GAAP firms with a sample of 428 IFRS reporters from 1990 through 2004. They also find similar financial reporting quality for 
IFRS and U.S. GAAP measures using the subsample of firms that are cross-listed on U.S. stock exchanges.

The extant International Accounting research literature examines the comparative information content of accounting numbers generated using alternative accounting principles before the advent of the EU 2005 wide-scale implementation of IFRS. An increasingly sizable body of related literature examines Form 20-F Item 17 or 18 reconciliations from non-U.S. practices to U.S. GAAP establishing a solid historical foundation for the interpretation of the value relevance of the Form 20-F reconciliations. In reviewing the extant research literature, Pownall and Schipper (1999) note that prior research documents significant differences between U.S. GAAP and both non-U.S. procedures and IFRS using Form 20-F reconciliation data and provides some indication that the differences are value-relevant. Amir, Harris, and Venuti (1993), for example, examine the value relevance of Form 20-F reconciling items between Non-U.S. domestic and U.S. GAAP earnings and shareholders' equity 1981-1991 using a sample of 101 cross-listed companies. Their research results indicate that Form 20-F reconciliations are equity share value relevant, both in aggregate and for certain specific components (e.g., property revaluations and capitalized goodwill). Providing only inconclusive evidence regarding the equity share value relevance of the Form 20-F reconciliations, Harris and Muller (1999) investigate just reconciliations of IFRS with U.S. GAAP for 31 companies from 1992 to 1996 and report (1) U.S. GAAP earnings Form 20-F reconciliation is value relevant and (2) U.S. GAAP is more highly associated with market variables after controlling for IFRS amounts in specific empirical statistical models.

More recent research related to comparative accounting principles measures examine the statistical properties of accounting measures for U.S. cross-listed companies employing a somewhat different perspective. Lang, Raedy, and Yetman (2006) compare U.S. cross-listed firms with non-cross-listed companies over the period of 1990 through 2001. Their results indicate that cross-listed firms' accounting measures display less earnings smoothing, increased timeliness for loss recognition, and greater share value-relevance than non-cross-listed companies. Lang, Raedy, and Wilson (2006) compares 131 U.S. cross-listed Form 20-F foreign private Level II or Level III ADR issuers firms with U.S. companies over the years 1991-2002. Their results indicate that U.S. GAAP accounting principles measurements for cross-listed firms differ from those of U.S. firms with respect to the time-series properties of reported earnings and accrual amounts, as well as the extent of the relation between accounting measures and equity share values. All things considered, the extant research literature indicates that differences in the reporting of U.S. cross-listed companies and U.S. companies exist even with the reconciliations. Empirical evidence from this literature suggests that cross-listed firms engage in less earnings management than non-cross-listed firms.

\section{Sample Selection Method and Sample Firms}

The sample for our research study is non-U.S. firms having ADRs listed on a major U.S. stock exchange and subject to the U.S. Securities and Exchange Commission periodic filing requirements. We are interested in ADR firms with SEC Form 20-F annual reporting requirements from January 1, 1990 and extending through December 31, 2015. The SEC requires that non-U.S. firms file their annual reports no later than six months after their fiscal year end, whereby calendar year-end firms file in months from March to July. We examine the accounting policy footnote for each 20-F filed with the SEC during this time period, in order to determine the accounting principles used to prepare the annual report included with the SEC Form 20-F filing. We collect the SEC Reporting GAAP, Net Income, and Shareholders Equity amounts from respective fiscal year-end Form 20-F filings. In order to obtain a list of ADR firms, we merge lists of ADR companies from Bank of New York and JP Morgan ADR Universe websites to identify ADRs traded on the NYSE, AMEX, or NASDAQ stock exchanges and are subject to SEC periodic filing requirements. The final sample results in 402 ADR firms reporting to the SEC using Home Country Accounting Principles, IFRS, and U.S. GAAP. The definitions of the variables employed in the statistical estimation and hypothesis tests and their computational measurement are listed and discussed below. 
Definition of variables employed in comparative statistical analyses of joint adr and equity share market response to earnings and SEC form 20-F releases

Variable Abbreviation $\quad$ Variable Definition and Computational Measurement

1. Annual Form 20-F Securities and Exchange Commission Filing:

$\begin{array}{ll}\text { Accounting } \quad \text { Principles } & \text { Identification of whether U.S.-listed ADRs use U.S. GAAP, IFRS (or predecessors), or Home Country } \\ \text { Choice Variable SEC Form } & \text { Accounting Standards in filing annual Form 20-F with the U.S. Securities and Exchange Commission. } \\ \text { 20-F GAAP } & \text { The determination of the accounting principles used for the SEC Form 20-F Filing was obtained through } \\ & \text { examination of Form 20-F on SEC EDGAR database (SEC.gov). To indicate increasing quality of } \\ & \text { accounting disclosures, the qualitative variable SEC 20-F GAAP } \text { it }_{\text {it }} \text { takes integer values 1, 2, 3. }\end{array}$

2. Daily ADR and Equity Share Returns and ADR and Equity Share Market Returns:

U.S. Exchange Listed ADR U.S. exchange-listed ADR share daily close price-to-close price dividend adjusted security returns Share Return $R_{i t}^{A D R}$ : (i.e., $R_{i t}^{A D R}=\frac{P_{i t}-P_{i t-1}+D_{i t}}{P_{i t-1}}$ for $\mathrm{i}^{\text {th }}$ U.S. exchange-listed ADR sample firm on trading day $\mathrm{t}$ ) from 2000 to 2015.

Home Country Equity Home country equity market daily close price-to-close price dividend adjusted security returns Share Return $R_{\text {it }}^{\text {Equity }}$ : (i.e., $R_{i t}^{\text {Equity }}=\Delta \gamma_{i t} \cdot \frac{P_{i t}^{\text {Equity }}-P_{i t-1}^{\text {Equity }}+\text { Dividend }_{t}^{\text {Equity }}}{P_{i t-1}^{\text {Equity }}}$ )for $\mathrm{i}^{\text {th }}$ U.S. exchange-listed ADR sample firm on trading day $\mathrm{t}$ after adjusting the equity share return for daily currency exchange rate changes $\left(\Delta \gamma_{i t}\right)$ from 2000 to 2015 .

U.S. Exchange Listed ADR Share Market Return $R_{M t}^{U S} N Y S E$ :

U.S. Exchange Listed ADR Equity Market Return $R_{M t}^{\text {Home }}$ :
Equal weighted average daily close price-to-close price dividend-adjusted security return over all firms

(having non-missing data) and NYSE from 2000 to 2015 (i.e., $R_{M t}^{\text {US NYSE }}=$

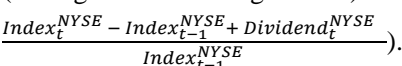

Equal weighted average daily close price-to-close price dividend-adjusted security return over all firms (having non-missing data) and over all firms comprising the major local market index for each sample firm local market NYSE from 2000 to 2015 (i.e., $R_{M t}^{\text {Home }}=\frac{\text { Index }_{t}^{\text {Home }}-\text { Index }_{t-1}^{\text {Home }}+\text { Dividend }_{t}^{\text {Home }}}{\text { Index }}$ ).

Earnings Release Date Qualitative Variable $D_{i t}^{E A D}$

$\begin{array}{lr}\text { Equity Share } & \text { Market } \\ \text { Reported } & \text { Earnings } \\ E_{\text {it }}^{\text {Reported }} & \end{array}$

3. Earnings Release Date, SEC Filing Date, Reported Earnings and US GAAP Earnings

Form 20-F Filing Date Qualitative Variable $D_{i t}^{S E C ~ 20-F}$

Form 20-F US GAAP Earnings $E_{i t}^{U S G A A P}$ earnings release date. $E_{i t}^{\text {Reported }}=\frac{E_{i t-1}^{\text {Reported }}}{P_{i t-3}^{A D R} \text { Equity }}=\frac{E_{i t-1}^{\text {Reported }}}{\gamma_{i t} \cdot \lambda_{i t} \cdot P_{i t-3}^{\text {Equity }}}$

Qualitative indicator variable taking a value of one on the day of the $\mathrm{i}^{\text {th }}$ sample firm earnings release, and valued at zero all other times $D_{i t}^{E A D}=\{0$ if trading day t is an earnings release date; 0 otherwise.

The U.S. dollar earnings per equity share (reported to the home country shareholders) divided by the home country equity share price expressed in U.S. dollars and in U.S. ADR share units three days preceding the

Qualitative indicator variable taking a value of one on the day of the $i^{\text {th }}$ sample firm SEC Form 20-F filing, and valued at zero all other times. $D_{i t}^{S E C} 20-F=\{0$ if trading day $\mathrm{t}$ is an SEC Form $20-\mathrm{F}$ filing date; 0 otherwise.

The U.S. dollar earnings per equity share computed in accordance with U.S. GAAP requirements (filed with the U.S. SEC Form 20-F) divided by the U.S. listed ADR share price three days preceding the U.S. SEC Form 20-F filing date. $E_{i t}^{U S G A A P}=\frac{E_{i t-1}^{U S G A P P}}{P_{i t-3}^{A D R}}$

\section{Daily ADR Home Country to U.S. Dollar Exchange Rate}

Daily Percentage Change Percentage change in the daily spot home currency to U.S. dollar exchange rate (i.e., $\% \Delta E R_{i t}=$

In Home Country $\left.\quad\left[\frac{E R_{i t}-E R_{i t-1}}{E R_{i t-1}}\right]\right)$.
Exchange Rate $\% \Delta \mathbf{E R}_{i t}:$

Descriptive statistics for the quantitative data variables employed in the statistical models are shown in Table 1. Table 1 shows distributional statistics for the data employed in the empirical analyses for the quantitative data variables used in this research. 
Table 1. Descriptive statistics U.S. listed ADR and security return

\begin{tabular}{|c|c|c|c|c|c|c|c|c|c|c|}
\hline \multicolumn{11}{|c|}{ Descriptive Statistics: } \\
\hline \multirow{6}{*}{ 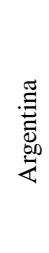 } & Variable & Mean & Std Dev & t Value & $\operatorname{Pr}>|\mathbf{t}|$ & $\mathbf{N}$ & Maximum & Minimum & Skewness & Kurtosis \\
\hline & $R_{i t}^{A D R}$ & 0.000455009 & 0.0305884 & 3.6 & 0.0003 & 58436 & 1 & -0.833505 & 3.315413 & 131.0708825 \\
\hline & $R_{i t}^{\text {Equity }}$ & 0.000583361 & 0.0295246 & 4.78 & $<.0001$ & 58436 & 1 & -0.656307 & 2.9290944 & 99.4411724 \\
\hline & $R_{M t}^{\text {Home }}$ & 0 & 0 & . & . & 58436 & 0 & 0 & . & . \\
\hline & $R_{M t}^{U S N Y S E}$ & 0.000234777 & 0.012471 & 4.55 & $<.0001$ & 58436 & 0.115307 & -0.089683 & -0.0189969 & 8.116037 \\
\hline & $R_{S t}^{F X}$ & 0 & 0 & . & . & 58436 & 0 & 0 & . & . \\
\hline \multirow{6}{*}{ 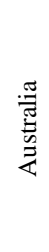 } & Variable & Mean & Std Dev & t Value & $\operatorname{Pr}>|t|$ & $\mathbf{N}$ & Maximum & Minimum & Skewness & Kurtosis \\
\hline & $R_{i t}^{A D R}$ & 0.000805249 & 0.0374714 & 4.81 & $<.0001$ & 50088 & 1 & -0.536269 & 3.9592178 & 90.3064042 \\
\hline & $R_{i t}^{\text {Equity }}$ & 0.000583418 & 0.0341991 & 3.82 & 0.0001 & 50088 & 1 & -0.603858 & 5.3923115 & 140.6334038 \\
\hline & $R_{M t}^{\text {Home }}$ & 0.000239622 & 0.0188122 & 2.85 & 0.0044 & 50088 & 0.154388 & -0.285585 & -2.0198891 & 32.744253 \\
\hline & $R_{M t}^{U S} N Y S E$ & 0.000234777 & 0.012471 & 4.21 & $<.0001$ & 50088 & 0.115307 & -0.089683 & -0.0189969 & 8.1161698 \\
\hline & $R_{S t}^{F X}$ & 0.000549251 & 0.0108064 & -11.38 & $<.0001$ & 50088 & 0.186735 & -0.289482 & -10.1766205 & 292.0105355 \\
\hline \multirow{6}{*}{$\frac{\Xi}{\vec{\Xi}_{0}}$} & Variable & Mean & Std Dev & t Value & $\operatorname{Pr}>|\mathbf{t}|$ & $\mathbf{N}$ & Maximum & Minimum & Skewness & Kurtosis \\
\hline & $R_{i t}^{A D R}$ & 0.000282039 & 0.0219092 & 0.83 & 0.4056 & 4174 & 0.685475 & -0.476987 & 5.5702961 & 292.021248 \\
\hline & $R_{i t}^{\text {Equity }}$ & 0.000283057 & 0.0171504 & 1.07 & 0.2864 & 4174 & 0.269371 & -0.138669 & 1.78638 & 35.7793094 \\
\hline & $R_{M t}^{\text {Home }}$ & 0.000405795 & 0.0147724 & 1.77 & 0.076 & 4174 & 0.087618 & -0.147464 & -0.655113 & 8.7917384 \\
\hline & $R_{M t}^{U S} N Y S E$ & 0.000234777 & 0.0124724 & 1.22 & 0.224 & 4174 & 0.115307 & -0.089683 & -0.0190032 & 8.1264083 \\
\hline & $R_{S t}^{F X}$ & 0.000059562 & 0.0084061 & 0.46 & 0.6471 & 4174 & 0.083107 & -0.083263 & -0.3928657 & 11.7425953 \\
\hline \multirow{6}{*}{$\begin{array}{l}\overline{\text { Tี }} \\
\text { صี }\end{array}$} & Variable & Mean & Std Dev & t Value & $\operatorname{Pr}>|t|$ & $\mathbf{N}$ & Maximum & Minimum & Skewness & Kurtosis \\
\hline & $R_{i t}^{A D R}$ & 0.000651238 & 0.025603 & 9 & $<.0001$ & 125220 & 0.746269 & -0.578125 & 0.6755475 & 28.7496864 \\
\hline & $R_{i t}^{\text {Equity }}$ & 0.000642469 & 0.0265835 & 8.55 & $<.0001$ & 125220 & 0.825 & -0.424658 & 0.7962812 & 30.0259184 \\
\hline & $R_{M t}^{\text {Home }}$ & 0.000369278 & 0.0135418 & 9.65 & $<.0001$ & 125220 & 0.102135 & -0.088928 & -0.0338238 & 5.382482 \\
\hline & $R_{M t}^{U S} N Y S E$ & 0.000234777 & 0.012471 & 6.66 & $<.0001$ & 125220 & 0.115307 & -0.089683 & -0.0189966 & 8.1156118 \\
\hline & $R_{S t}^{F X}$ & 0.000091999 & 0.0045397 & 7.17 & $<.0001$ & 125220 & 0.023474 & -0.025053 & 0.1776906 & 4.0216514 \\
\hline \multirow{6}{*}{$\stackrel{\stackrel{\Xi}{ت}}{\ddot{U}}$} & Variable & Mean & Std Dev & t Value & $\operatorname{Pr}>|t|$ & $\mathbf{N}$ & Maximum & Minimum & Skewness & Kurtosis \\
\hline & $R_{i t}^{A D R}$ & 0.000462465 & 0.0174741 & 6.62 & $<.0001$ & 62610 & 0.428571 & -0.25 & 0.809993 & 35.0208953 \\
\hline & $R_{i t}^{\text {Equity }}$ & 0.000419986 & 0.0193298 & 5.44 & $<.0001$ & 62610 & 1 & -0.901042 & 2.0138175 & 235.9909266 \\
\hline & $R_{M t}^{\text {Home }}$ & 0.000529905 & 0.0136185 & 9.74 & $<.0001$ & 62610 & 0.114902 & -0.094447 & -0.014328 & 5.7536119 \\
\hline & $R_{M t}^{U S N Y S E}$ & 0.000234777 & 0.012471 & 4.71 & $<.0001$ & 62610 & 0.115307 & -0.089683 & -0.0189968 & 8.1159838 \\
\hline & $R_{S t}^{F X}$ & -0.000129376 & 0.010669 & -3.03 & 0.0024 & 62610 & 0.116078 & -0.080456 & 0.0770755 & 9.0781837 \\
\hline \multirow{6}{*}{$\stackrel{\widetilde{J}}{\stackrel{\Xi}{\Xi}}$} & Variable & Mean & Std Dev & t Value & $\operatorname{Pr}>|\mathbf{t}|$ & $\mathbf{N}$ & Maximum & Minimum & Skewness & Kurtosis \\
\hline & $R_{i t}^{A D R}$ & 0.00023581 & 0.0230301 & 5.25 & $<.0001$ & 262962 & 1 & -0.559026 & 2.2757898 & 88.2708772 \\
\hline & $R_{i t}^{\text {Equity }}$ & 0.000314166 & 0.0241488 & 6.67 & $<.0001$ & 262962 & 1 & -0.499511 & 1.8839607 & 61.7360857 \\
\hline & $R_{M t}^{\text {Home }}$ & 0.000339668 & 0.0112937 & 15.42 & $<.0001$ & 262962 & 0.127876 & -0.100039 & -0.215442 & 10.0485856 \\
\hline & $R_{M t}^{U S N Y S E}$ & 0.000234777 & 0.0124709 & 9.65 & $<.0001$ & 262962 & 0.115307 & -0.089683 & -0.0189965 & 8.115417 \\
\hline & $R_{S t}^{F X}$ & 0.000050016 & 0.0064283 & -3.99 & $<.0001$ & 262962 & 0.044459 & -0.046512 & -0.1771774 & 4.092336 \\
\hline \multirow{6}{*}{$\frac{\cdot \frac{\pi}{0}}{\frac{0}{0}}$} & Variable & Mean & Std Dev & t Value & $\operatorname{Pr}>|\mathbf{t}|$ & $\mathbf{N}$ & Maximum & Minimum & Skewness & Kurtosis \\
\hline & $R_{i t}^{A D R}$ & 0.000898799 & 0.0268706 & 3.06 & 0.0022 & 8348 & 0.5 & -0.4 & 2.0855142 & 60.6637898 \\
\hline & $R_{i t}^{\text {Equity }}$ & 0.000659926 & 0.0207097 & 2.91 & 0.0036 & 8348 & 0.303058 & -0.302627 & 0.419978 & 34.5238676 \\
\hline & $R_{M t}^{\text {Home }}$ & 0.000734666 & 0.0186562 & 3.6 & 0.0003 & 8348 & 0.170284 & -0.126331 & 0.4427062 & 8.2282359 \\
\hline & $R_{M t}^{U S N Y S E}$ & 0.000234777 & 0.0124717 & 1.72 & 0.0855 & 8348 & 0.115307 & -0.089683 & -0.0189998 & 8.1208216 \\
\hline & $R_{S t}^{F X}$ & 0.000058725 & 0.0010496 & 5.11 & $<.0001$ & 8348 & 0.019994 & -0.018194 & 0.1454048 & 62.0566258 \\
\hline \multirow{6}{*}{ 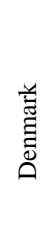 } & Variable & Mean & Std Dev & t Value & $\operatorname{Pr}>|t|$ & $\mathbf{N}$ & Maximum & Minimum & Skewness & Kurtosis \\
\hline & $R_{i t}^{A D R}$ & 0.000713533 & 0.0172026 & 2.68 & 0.0074 & 4174 & 0.155328 & -0.21112 & -0.4094812 & 15.2169618 \\
\hline & $R_{i t}^{\text {Equity }}$ & 0.000714735 & 0.0168891 & 2.73 & 0.0063 & 4174 & 0.172171 & -0.18889 & 0.1887495 & 13.5992642 \\
\hline & $R_{M t}^{\text {Home }}$ & 0.000665613 & 0.0098641 & 4.36 & $<.0001$ & 4174 & 0.123462 & -0.081445 & -0.1070604 & 15.4235838 \\
\hline & $R_{M t}^{U S} N Y S E$ & 0.000234777 & 0.0124724 & 1.22 & 0.224 & 4174 & 0.115307 & -0.089683 & -0.0190032 & 8.1264083 \\
\hline & $R_{S t}^{F X}$ & -0.000100046 & 0.0070607 & -0.92 & 0.36 & 4174 & 0.1145 & -0.08046 & 0.6101843 & 27.6728761 \\
\hline \multirow{6}{*}{$\underset{\mathrm{I}}{\stackrel{\vec{\Xi}}{\Xi}}$} & Variable & Mean & Std Dev & t Value & $\operatorname{Pr}>|t|$ & $\mathbf{N}$ & Maximum & Minimum & Skewness & Kurtosis \\
\hline & $R_{i t}^{A D R}$ & 0.000152278 & 0.0227898 & 0.61 & 0.5415 & 8348 & 0.360059 & -0.224444 & 0.4481832 & 18.753983 \\
\hline & $R_{i t}^{\text {Equity }}$ & 0.000111695 & 0.0220653 & 0.46 & 0.6437 & 8348 & 0.195397 & -0.253696 & -0.1331996 & 12.1939958 \\
\hline & $R_{M t}^{\text {Home }}$ & 0.000484887 & 0.0139132 & 3.18 & 0.0015 & 8348 & 0.118225 & -0.128892 & -0.1518113 & 7.152804 \\
\hline & $R_{M t}^{U S N Y S E}$ & 0.000234777 & 0.0124717 & 1.72 & 0.0855 & 8348 & 0.115307 & -0.089683 & -0.0189998 & 8.1208216 \\
\hline & $R_{S t}^{F X}$ & 0.000033568 & 0.0064382 & 0.48 & 0.6338 & 8348 & 0.035161 & -0.026011 & 0.1260128 & 1.5804671 \\
\hline
\end{tabular}




\begin{tabular}{|c|c|c|c|c|c|c|c|c|c|c|}
\hline \multirow{6}{*}{$\begin{array}{l}\ddot{U} \\
\text { : } \\
\text { : }\end{array}$} & Variable & Mean & Std Dev & t Value & $\operatorname{Pr}>|\mathbf{t}|$ & $\mathbf{N}$ & Maximum & Minimum & Skewness & Kurtosis \\
\hline & $R_{i t}^{A D R}$ & 0.000466913 & 0.0316445 & 3.16 & 0.0016 & 45914 & 1 & -0.501605 & 3.2621006 & 89.0254267 \\
\hline & $R_{i t}^{\text {Equity }}$ & 0.000408453 & 0.0301298 & 2.9 & 0.0037 & 45914 & 1 & -0.501605 & 2.3639697 & 60.8847077 \\
\hline & $R_{M t}^{\text {Home }}$ & 0.00019245 & 0.0198602 & 2.08 & 0.0379 & 45914 & 0.154752 & -0.169449 & -0.1019721 & 6.1699137 \\
\hline & $R_{M t}^{U S N Y S E}$ & 0.000234777 & 0.012471 & 4.03 & $<.0001$ & 45914 & 0.115307 & -0.089683 & -0.018997 & 8.1162544 \\
\hline & $R_{S t}^{F X}$ & 0.000092359 & 0.0045246 & 4.37 & $<.0001$ & 45914 & 0.023562 & -0.025079 & 0.1251854 & 3.9895986 \\
\hline \multirow{6}{*}{ 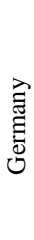 } & Variable & Mean & Std Dev & t Value & $\operatorname{Pr}>|t|$ & $\mathbf{N}$ & Maximum & Minimum & Skewness & Kurtosis \\
\hline & $R_{i t}^{A D R}$ & 0.000310818 & 0.0313978 & 2.22 & 0.0267 & 50088 & 1 & -0.624586 & 5.6478717 & 185.1335856 \\
\hline & $R_{i t}^{\text {Equity }}$ & 0.000237914 & 0.0267991 & 1.99 & 0.0469 & 50088 & 1 & -0.584615 & 3.6364774 & 138.4995589 \\
\hline & $R_{M t}^{\text {Home }}$ & 0.00026348 & 0.0149383 & 3.95 & $<.0001$ & 50088 & 0.112573 & -0.101261 & 0.1179609 & 5.7559367 \\
\hline & $R_{M t}^{U S N Y S E}$ & 0.000234777 & 0.012471 & 4.21 & $<.0001$ & 50088 & 0.115307 & -0.089683 & -0.0189969 & 8.1161698 \\
\hline & $R_{S t}^{F X}$ & 0.000092414 & 0.0045236 & 4.57 & $<.0001$ & 50088 & 0.0237 & -0.025076 & 0.1211433 & 4.0175398 \\
\hline \multirow{6}{*}{ 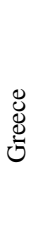 } & Variable & Mean & Std Dev & t Value & $\operatorname{Pr}>|t|$ & $\mathbf{N}$ & Maximum & Minimum & Skewness & Kurtosis \\
\hline & $R_{i t}^{A D R}$ & 0.000120647 & 0.0212024 & 0.74 & 0.4622 & 16696 & 0.198017 & -0.226727 & 0.0726643 & 12.2972245 \\
\hline & $R_{i t}^{\text {Equity }}$ & 0.000174148 & 0.0206888 & 1.09 & 0.2768 & 16696 & 0.604369 & -0.405376 & 1.6623477 & 78.4916775 \\
\hline & $R_{M t}^{\text {Home }}$ & 0.000255365 & 0.0143549 & 2.3 & 0.0215 & 16696 & 0.176765 & -0.082462 & 0.3246166 & 9.3460889 \\
\hline & $R_{M t}^{U S N Y S E}$ & 0.000234777 & 0.0124713 & 2.43 & 0.015 & 16696 & 0.115307 & -0.089683 & -0.0189981 & 8.1180301 \\
\hline & $R_{S t}^{F X}$ & 0.000092356 & 0.0045243 & 2.64 & 0.0084 & 16696 & 0.02394 & -0.025067 & 0.131403 & 4.0004596 \\
\hline \multirow{6}{*}{ 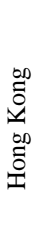 } & Variable & Mean & Std Dev & t Value & $\operatorname{Pr}>|\mathbf{t}|$ & $\mathbf{N}$ & Maximum & Minimum & Skewness & Kurtosis \\
\hline & $R_{i t}^{A D R}$ & 0.000157866 & 0.0379151 & 0.71 & 0.4767 & 29218 & 1 & -0.666667 & 5.8757176 & 176.8180642 \\
\hline & $R_{i t}^{\text {Equity }}$ & 0.000211718 & 0.038532 & 0.94 & 0.3476 & 29218 & 1 & -0.666667 & 5.5965421 & 164.0305349 \\
\hline & $R_{M t}^{\text {Home }}$ & -0.000388383 & 0.020634 & -3.22 & 0.0013 & 29218 & 0.146595 & -0.195661 & -0.0729804 & 6.9284879 \\
\hline & $R_{M t}^{U S N Y S E}$ & 0.000234777 & 0.0124711 & 3.22 & 0.0013 & 29218 & 0.115307 & -0.089683 & -0.0189974 & 8.1168342 \\
\hline & $R_{S t}^{F X}$ & 0.000109304 & 0.0088901 & 2.1 & 0.0356 & 29218 & 0.413592 & -0.193708 & 26.3150576 & 1299.54 \\
\hline \multirow{6}{*}{ 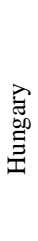 } & Variable & Mean & Std Dev & t Value & $\operatorname{Pr}>|t|$ & $\mathbf{N}$ & Maximum & Minimum & Skewness & Kurtosis \\
\hline & $R_{i t}^{A D R}$ & 0.000101329 & 0.0195201 & 0.34 & 0.7374 & 4174 & 0.172414 & -0.154762 & 0.3413545 & 7.6866264 \\
\hline & $R_{i t}^{\text {Equity }}$ & 0.000125725 & 0.0197917 & 0.41 & 0.6815 & 4174 & 0.153634 & -0.158024 & -0.1324034 & 7.6056464 \\
\hline & $R_{M t}^{\text {Home }}$ & 0.000294867 & 0.0138189 & 1.38 & 0.1681 & 4174 & 0.111937 & -0.109176 & -0.0834334 & 6.897782 \\
\hline & $R_{M t}^{U S N Y S E}$ & 0.000234777 & 0.0124724 & 1.22 & 0.224 & 4174 & 0.115307 & -0.089683 & -0.0190032 & 8.1264083 \\
\hline & $R_{S t}^{F X}$ & $8.60 \mathrm{E}-07$ & 0.000362466 & 0.15 & 0.8782 & 4174 & 0.007613 & -0.00744 & 1.9263839 & 121.0717053 \\
\hline \multirow{6}{*}{ 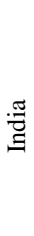 } & Variable & Mean & Std Dev & t Value & $\operatorname{Pr}>|\mathbf{t}|$ & $\mathbf{N}$ & Maximum & Minimum & Skewness & Kurtosis \\
\hline & $R_{i t}^{A D R}$ & 0.000624389 & 0.032317 & 4.67 & $<.0001$ & 58436 & 1 & -0.777471 & 3.4100609 & 97.4347341 \\
\hline & $R_{i t}^{\text {Equity }}$ & 0.000498824 & 0.0319307 & 3.78 & 0.0002 & 58436 & 1 & -0.843823 & 3.5714249 & 104.9103139 \\
\hline & $R_{M t}^{\text {Home }}$ & 0.000203132 & 0.014669 & 3.35 & 0.0008 & 58436 & 0.14086 & -0.119528 & 0.1527443 & 7.4419555 \\
\hline & $R_{M t}^{U S N Y S E}$ & 0.000234777 & 0.012471 & 4.55 & $<.0001$ & 58436 & 0.115307 & -0.089683 & -0.0189969 & 8.116037 \\
\hline & $R_{S t}^{F X}$ & $7.08 \mathrm{E}-06$ & 0.0094923 & 0.18 & 0.8569 & 58436 & 0.06645 & -0.075783 & -0.2687272 & 4.2024679 \\
\hline \multirow{6}{*}{ 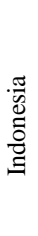 } & Variable & Mean & Std Dev & t Value & $\operatorname{Pr}>|\mathbf{t}|$ & $\mathbf{N}$ & Maximum & Minimum & Skewness & Kurtosis \\
\hline & $R_{i t}^{A D R}$ & 0.0032399 & 0.0835426 & 3.54 & 0.0004 & 8348 & 1 & -0.5 & 6.1684913 & 84.7355226 \\
\hline & $R_{i t}^{\text {Equity }}$ & 0.000537775 & 0.0223457 & 2.2 & 0.0279 & 8348 & 0.260537 & -0.202603 & 0.5114889 & 13.5420457 \\
\hline & $R_{M t}^{H o m e}$ & 0.000538213 & 0.0149922 & 3.28 & 0.001 & 8348 & 0.162804 & -0.118246 & -0.2981035 & 8.5718802 \\
\hline & $R_{M t}^{U S N Y S E}$ & 0.000234777 & 0.0124717 & 1.72 & 0.0855 & 8348 & 0.115307 & -0.089683 & -0.0189998 & 8.1208216 \\
\hline & $R_{S t}^{F X}$ & -0.000091223 & 0.0044079 & -1.89 & 0.0587 & 8348 & 0.037125 & -0.05096 & -0.347528 & 12.8349358 \\
\hline \multirow{6}{*}{ 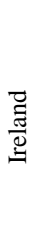 } & Variable & Mean & Std Dev & t Value & $\operatorname{Pr}>|\mathbf{t}|$ & $\mathbf{N}$ & Maximum & Minimum & Skewness & Kurtosis \\
\hline & $R_{i t}^{A D R}$ & 0.000470725 & 0.0337672 & 2.7 & 0.0069 & 37566 & 0.882646 & -0.678277 & 1.3187061 & 65.4685125 \\
\hline & $R_{i t}^{\text {Equity }}$ & 0.000415259 & 0.0318541 & 2.53 & 0.0115 & 37566 & 0.6 & -0.7026 & 0.3214383 & 45.7073386 \\
\hline & $R_{M t}^{\text {Home }}$ & 0.000431599 & 0.0180607 & 4.63 & $<.0001$ & 37566 & 0.144527 & -0.149823 & -0.3117978 & 7.1423139 \\
\hline & $R_{M t}^{U S N Y S E}$ & 0.000234777 & 0.0124711 & 3.65 & 0.0003 & 37566 & 0.115307 & -0.089683 & -0.0189971 & 8.1164798 \\
\hline & $R_{S t}^{F X}$ & -0.000138711 & 0.0065921 & -4.08 & $<.0001$ & 37566 & 0.093948 & -0.078195 & 0.2189548 & 29.8025015 \\
\hline \multirow{6}{*}{$\begin{array}{l}\overrightarrow{\mathbb{E}} \\
\ddot{\omega}\end{array}$} & Variable & Mean & Std Dev & t Value & $\operatorname{Pr}>|t|$ & $\mathbf{N}$ & Maximum & Minimum & Skewness & Kurtosis \\
\hline & $R_{i t}^{A D R}$ & 0.000412094 & 0.0263777 & 2.26 & 0.024 & 20870 & 1 & -0.935484 & 3.457637 & 272.2196744 \\
\hline & $R_{i t}^{\text {Equity }}$ & 0.000530057 & 0.0308647 & 2.48 & 0.0131 & 20870 & 1 & -0.944188 & 3.0465772 & 194.9435807 \\
\hline & $R_{M t}^{\text {Home }}$ & 0.000303819 & 0.0150447 & 2.92 & 0.0035 & 20870 & 0.09793 & -0.135224 & -0.3980175 & 6.3982573 \\
\hline & $R_{M t}^{U S} N Y S E$ & 0.000234777 & 0.0124712 & 2.72 & 0.0065 & 20870 & 0.115307 & -0.089683 & -0.0189977 & 8.117472 \\
\hline & $R_{S t}^{F X}$ & 0.00009235 & 0.0045317 & 2.94 & 0.0032 & 20870 & 0.024871 & -0.026492 & 0.1447584 & 4.1744864 \\
\hline \multirow{6}{*}{ 䔍 } & Variable & Mean & Std Dev & t Value & $\operatorname{Pr}>|t|$ & $\mathbf{N}$ & Maximum & Minimum & Skewness & Kurtosis \\
\hline & $R_{i t}^{A D R}$ & 0.000210745 & 0.024474 & 1.67 & 0.0951 & 37566 & 0.884398 & -0.558625 & 3.7044884 & 134.2370801 \\
\hline & $R_{i t}^{\text {Equity }}$ & 0.000284153 & 0.0248198 & 2.22 & 0.0265 & 37566 & 1 & -0.959949 & 1.7450962 & 286.6413386 \\
\hline & $R_{M t}^{\text {Home }}$ & 0.000305832 & 0.0110266 & 5.38 & $<.0001$ & 37566 & 0.071337 & -0.076895 & -0.3182325 & 4.0633009 \\
\hline & $R_{M t}^{U S N Y S E}$ & 0.000234777 & 0.0124711 & 3.65 & 0.0003 & 37566 & 0.115307 & -0.089683 & -0.0189971 & 8.1164798 \\
\hline & $R_{S t}^{F X}$ & 0.000025423 & 0.005024 & 0.98 & 0.3267 & 37566 & 0.030191 & -0.0405 & -0.2034434 & 5.4533928 \\
\hline
\end{tabular}




\begin{tabular}{|c|c|c|c|c|c|c|c|c|c|c|}
\hline \multirow{6}{*}{ 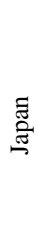 } & Variable & Mean & Std Dev & t Value & $\operatorname{Pr}>|t|$ & $\mathbf{N}$ & Maximum & Minimum & Skewness & Kurtosis \\
\hline & $R_{i t}^{A D R}$ & 0.00017807 & 0.0218439 & 2.53 & 0.0115 & 96002 & 0.438298 & -0.31134 & 0.5581564 & 13.2808492 \\
\hline & $R_{i t}^{\text {Equity }}$ & 0.000217611 & 0.0224322 & 3.01 & 0.0027 & 96002 & 1 & -0.60254 & 2.9299708 & 108.0377759 \\
\hline & $R_{M t}^{\text {Home }}$ & 0.000190566 & 0.0155969 & 3.79 & 0.0002 & 96002 & 0.119465 & -0.103056 & 0.0413117 & 5.6409099 \\
\hline & $R_{M t}^{U S N Y S E}$ & 0.000234777 & 0.012471 & 5.83 & $<.0001$ & 96002 & 0.115307 & -0.089683 & -0.0189967 & 8.1157251 \\
\hline & $R_{S t}^{F X}$ & 0.000093152 & 0.0046773 & 6.17 & $<.0001$ & 96002 & 0.051836 & -0.036961 & 0.3411121 & 8.0003156 \\
\hline \multirow{7}{*}{$\begin{array}{l}\mathscr{D} \\
\stackrel{0}{0}\end{array}$} & Variable & Mean & Std Dev & t Value & $\operatorname{Pr}>|t|$ & $\mathbf{N}$ & Maximum & Minimum & Skewness & Kurtosis \\
\hline & $R_{i t}^{A D R}$ & 0.000716237 & 0.0394775 & 3.89 & 0.0001 & 45914 & 1 & -0.863636 & 5.0725227 & 153.4307217 \\
\hline & $R_{i t}^{\text {Equity }}$ & 0.000438909 & 0.0322503 & 2.92 & 0.0035 & 45914 & 1 & -0.863636 & 5.3400244 & 203.879501 \\
\hline & $R_{M t}^{\text {Home }}$ & 0 & 0 & & & 45914 & 0 & 0 & . & \\
\hline & $R_{M t}^{U S N Y S E}$ & 0.000234777 & 0.012471 & 4.03 & $<.0001$ & 45914 & 0.115307 & -0.089683 & -0.018997 & 8.1162544 \\
\hline & $R_{S t}^{F X}$ & 0 & 0 & & & 45914 & 0 & 0 & . & \\
\hline & Variable & Mean & Std Dev & t Value & $\operatorname{Pr}>|t|$ & $\mathbf{N}$ & Maximum & Minimum & Skewness & Kurtosis \\
\hline \multirow{5}{*}{ 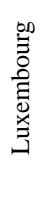 } & $R_{i t}^{A D R}$ & 0.000734905 & 0.0315077 & 1.51 & 0.1319 & 4174 & 0.244131 & -0.206272 & 0.5873344 & 8.7869323 \\
\hline & $R_{i t}^{\text {Equity }}$ & 0.000861408 & 0.0329785 & 1.69 & 0.0916 & 4174 & 0.539683 & -0.239691 & 1.5076897 & 26.7262817 \\
\hline & $R_{M t}^{\text {Home }}$ & 0.000384348 & 0.0197163 & 1.26 & 0.2079 & 4174 & 0.279704 & -0.184005 & 0.1629167 & 15.0486932 \\
\hline & $R_{M t}^{U S N Y S E}$ & 0.000234777 & 0.0124724 & 1.22 & 0.224 & 4174 & 0.115307 & -0.089683 & -0.0190032 & 8.1264083 \\
\hline & $R_{S t}^{F X}$ & 0.000022536 & 0.0082187 & 0.18 & 0.8594 & 4174 & 0.125 & -0.074074 & 1.3582071 & 29.9425916 \\
\hline \multirow{7}{*}{ 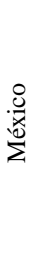 } & Variable & Mean & Std Dev & t Value & $\operatorname{Pr}>|t|$ & $\mathbf{N}$ & Maximum & Minimum & Skewness & Kurtosis \\
\hline & $R_{i t}^{A D R}$ & 0.000379402 & 0.0209076 & 5.5 & $<.0001$ & 91828 & 1 & -0.577508 & 3.3658138 & 203.343515 \\
\hline & $R_{i t}^{\text {Equity }}$ & 0.000386148 & 0.0217526 & 5.38 & $<.0001$ & 91828 & 1 & -0.808287 & 2.6262716 & 150.2589404 \\
\hline & $R_{M t}^{\text {Home }}$ & 0.000247278 & 0.0104252 & 7.19 & $<.0001$ & 91828 & 0.10676 & -0.06585 & 0.0562797 & 7.3763933 \\
\hline & $R_{M t}^{U S N Y S E}$ & 0.000234777 & 0.012471 & 5.7 & $<.0001$ & 91828 & 0.115307 & -0.089683 & -0.0189967 & 8.1157471 \\
\hline & $R_{S t}^{F X}$ & 0 & 0 & & & 91828 & 0 & 0 & 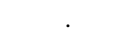 & \\
\hline & Variable & Mean & Std Dev & t Value & $\operatorname{Pr}>|t|$ & $\mathbf{N}$ & Maximum & Minimum & Skewness & Kurtosis \\
\hline \multirow{5}{*}{ 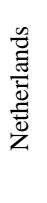 } & $R_{i t}^{A D R}$ & 0.000309573 & 0.0254984 & 2.22 & 0.0265 & 33392 & 0.892239 & -0.405304 & 3.4583572 & 101.1640174 \\
\hline & $R_{i t}^{\text {Equity }}$ & 0.000300327 & 0.0248498 & 2.21 & 0.0272 & 33392 & 0.62212 & -0.27491 & 1.5389663 & 45.318028 \\
\hline & $R_{M t}^{\text {Home }}$ & 0.00045293 & 0.0144427 & 5.73 & $<.0001$ & 33392 & 0.141915 & -0.094623 & 0.0596555 & 7.0655687 \\
\hline & $R_{M t}^{U S N Y S E}$ & 0.000234777 & 0.0124711 & 3.44 & 0.0006 & 33392 & 0.115307 & -0.089683 & -0.0189972 & 8.1166349 \\
\hline & $R_{S t}^{F X}$ & -0.000118297 & 0.0071956 & -3 & 0.0027 & 33392 & 0.07689 & -0.083467 & -0.5398489 & 13.4542197 \\
\hline \multirow{6}{*}{ 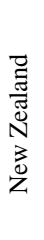 } & Variable & Mean & Std Dev & t Value & $\operatorname{Pr}>|t|$ & $\mathbf{N}$ & Maximum & Minimum & Skewness & Kurtosis \\
\hline & $R_{i t}^{A D R}$ & 0.00009312 & 0.0159227 & 0.38 & 0.7056 & 4174 & 0.102272 & -0.108622 & -0.3088065 & 5.313642 \\
\hline & $R_{i t}^{\text {Equity }}$ & 0.000153971 & 0.016652 & 0.6 & 0.5503 & 4174 & 0.141076 & -0.157381 & -0.0801423 & 10.409192 \\
\hline & $R_{M t}^{\text {Home }}$ & 0.000198692 & 0.0146255 & 0.88 & 0.3802 & 4174 & 0.107607 & -0.10832 & -0.0454062 & 6.8210638 \\
\hline & $R_{M t}^{U S N Y S E}$ & 0.000234777 & 0.0124724 & 1.22 & 0.224 & 4174 & 0.115307 & -0.089683 & -0.0190032 & 8.1264083 \\
\hline & $R_{S t}^{F X}$ & 0.000099901 & 0.0059757 & 1.08 & 0.2802 & 4174 & 0.187359 & -0.159075 & 2.8598485 & 352.3129704 \\
\hline \multirow{6}{*}{$\begin{array}{l}\text { ते } \\
\text { ì } \\
\text { Zे }\end{array}$} & Variable & Mean & Std Dev & t Value & $\operatorname{Pr}>|t|$ & $\mathbf{N}$ & Maximum & Minimum & Skewness & Kurtosis \\
\hline & $R_{i t}^{A D R}$ & 0.00055094 & 0.0190699 & 2.64 & 0.0083 & 8348 & 0.171078 & -0.237448 & -0.0763978 & 10.9429826 \\
\hline & $R_{i t}^{\text {Equity }}$ & 0.00056525 & 0.0203149 & 2.54 & 0.011 & 8348 & 0.163121 & -0.279162 & -0.15348 & 12.4327086 \\
\hline & $R_{M t}^{\text {Home }}$ & 0.000429117 & 0.0113977 & 3.44 & 0.0006 & 8348 & 0.097301 & -0.083109 & -0.4478829 & 6.165403 \\
\hline & $R_{M t}^{U S N Y S E}$ & 0.000234777 & 0.0124717 & 1.72 & 0.0855 & 8348 & 0.115307 & -0.089683 & -0.0189998 & 8.1208216 \\
\hline & $R_{S t}^{F X}$ & 0.000098734 & 0.0084718 & 1.06 & 0.287 & 8348 & 0.061439 & -0.066488 & -0.3190851 & 3.2791209 \\
\hline \multirow{6}{*}{ 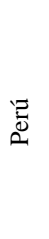 } & Variable & Mean & Std Dev & t Value & $\operatorname{Pr}>|t|$ & $\mathbf{N}$ & Maximum & Minimum & Skewness & Kurtosis \\
\hline & $R_{i t}^{A D R}$ & 0.000914308 & 0.0239239 & 2.47 & 0.0136 & 4174 & 0.229868 & -0.195885 & -0.1610403 & 11.8186627 \\
\hline & $R_{i t}^{\text {Equity }}$ & 0.000955789 & 0.0246169 & 2.51 & 0.0122 & 4174 & 0.184448 & -0.194411 & 0.0129747 & 8.1495176 \\
\hline & $R_{M t}^{\text {Home }}$ & 0.000409622 & 0.0181495 & 1.46 & 0.1449 & 4174 & 0.149136 & -0.126884 & -0.2106927 & 6.6074467 \\
\hline & $R_{M t}^{U S N Y S E}$ & 0.000234777 & 0.0124724 & 1.22 & 0.224 & 4174 & 0.115307 & -0.089683 & -0.0190032 & 8.1264083 \\
\hline & $R_{S t}^{F X}$ & 4.24E-06 & 0.0079072 & 0.03 & 0.9724 & 4174 & 0.056563 & -0.047035 & -0.0676188 & 3.1234335 \\
\hline \multirow{6}{*}{ 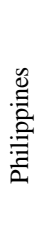 } & Variable & Mean & Std Dev & t Value & $\operatorname{Pr}>|t|$ & $\mathbf{N}$ & Maximum & Minimum & Skewness & Kurtosis \\
\hline & $R_{i t}^{A D R}$ & 0.000234787 & 0.0178087 & 1.2 & 0.2284 & 8348 & 0.657061 & -0.313892 & 6.3794498 & 262.6195882 \\
\hline & $R_{i t}^{\text {Equity }}$ & 0.00019285 & 0.0172083 & 1.02 & 0.3059 & 8348 & 0.657061 & -0.313892 & 6.4408479 & 285.7343859 \\
\hline & $R_{M t}^{\text {Home }}$ & 0.000468324 & 0.0089649 & 4.77 & $<.0001$ & 8348 & 0.072022 & -0.08128 & -0.738119 & 11.6487171 \\
\hline & $R_{M t}^{U S N Y S E}$ & 0.000234777 & 0.0124717 & 1.72 & 0.0855 & 8348 & 0.115307 & -0.089683 & -0.0189998 & 8.1208216 \\
\hline & $R_{S t}^{F X}$ & 0.000012268 & 0.0031373 & 0.36 & 0.7209 & 8348 & 0.033777 & -0.031663 & -0.1079539 & 15.3586677 \\
\hline \multirow{6}{*}{$\begin{array}{l}\bar{F} \\
\text { 㟧 } \\
\text { 总 }\end{array}$} & Variable & Mean & Std Dev & t Value & $\operatorname{Pr}>|t|$ & $\mathbf{N}$ & Maximum & Minimum & Skewness & Kurtosis \\
\hline & $R_{i t}^{A D R}$ & 0.000348176 & 0.0175703 & 1.28 & 0.2005 & 4174 & 0.209677 & -0.17654 & 0.9891086 & 20.1790507 \\
\hline & $R_{i t}^{\text {Equity }}$ & 0.000313464 & 0.0185408 & 1.09 & 0.2748 & 4174 & 0.176331 & -0.132594 & 0.5039093 & 9.9904016 \\
\hline & $R_{M t}^{\text {Home }}$ & 0.000377991 & 0.0132585 & 1.84 & 0.0656 & 4174 & 0.215956 & -0.113189 & 1.1765839 & 26.8259339 \\
\hline & $R_{M t}^{U S N Y S E}$ & 0.000234777 & 0.0124724 & 1.22 & 0.224 & 4174 & 0.115307 & -0.089683 & -0.0190032 & 8.1264083 \\
\hline & $R_{S t}^{F X}$ & 0 & 0 & . & & 4174 & 0 & 0 & . & \\
\hline
\end{tabular}




\begin{tabular}{|c|c|c|c|c|c|c|c|c|c|c|}
\hline \multirow{6}{*}{ 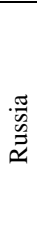 } & Variable & Mean & Std Dev & t Value & $\operatorname{Pr}>|t|$ & $\mathbf{N}$ & Maximum & Minimum & Skewness & Kurtosis \\
\hline & $R_{i t}^{A D R}$ & 0.000652126 & 0.0218788 & 4.31 & $<.0001$ & 20870 & 1 & -0.571323 & 7.0843942 & 312.5076699 \\
\hline & $R_{i t}^{\text {Equity }}$ & 0.000858572 & 0.0308829 & 4.02 & $<.0001$ & 20870 & 0.602425 & -0.376123 & 1.9720307 & 47.5473775 \\
\hline & $R_{M t}^{\text {Home }}$ & 0.000100705 & 0.0135023 & 1.08 & 0.2813 & 20870 & 0.11543 & -0.120198 & -0.0474811 & 7.0051504 \\
\hline & $R_{M t}^{U S N Y S E}$ & 0.000234777 & 0.0124712 & 2.72 & 0.0065 & 20870 & 0.115307 & -0.089683 & -0.0189977 & 8.117472 \\
\hline & $R_{S t}^{F X}$ & 0.000125052 & 0.0094585 & 1.91 & 0.0561 & 20870 & 0.430515 & -0.306604 & 14.4601852 & 1291.02 \\
\hline \multirow{6}{*}{ 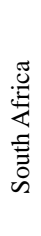 } & Variable & Mean & Std Dev & t Value & $\operatorname{Pr}>|t|$ & $\mathbf{N}$ & Maximum & Minimum & Skewness & Kurtosis \\
\hline & $R_{i t}^{A D R}$ & 0.000632865 & 0.0257916 & 4.48 & $<.0001$ & 33392 & 0.371904 & -0.267784 & 0.687853 & 13.077314 \\
\hline & $R_{i t}^{\text {Equity }}$ & 0.000668717 & 0.0273211 & 4.47 & $<.0001$ & 33392 & 0.504851 & -0.336192 & 0.9287036 & 19.4493298 \\
\hline & $R_{M t}^{\text {Home }}$ & 0.000250184 & 0.0116058 & 3.94 & $<.0001$ & 33392 & 0.097753 & -0.081812 & -0.2170346 & 5.832093 \\
\hline & $R_{M t}^{U S N Y S E}$ & 0.000234777 & 0.0124711 & 3.44 & 0.0006 & 33392 & 0.115307 & -0.089683 & -0.0189972 & 8.1166349 \\
\hline & $R_{S t}^{F X}$ & 0.000042737 & 0.0033158 & 2.36 & 0.0185 & 33392 & 0.023507 & -0.026326 & -0.041864 & 5.0331045 \\
\hline \multirow{6}{*}{$\begin{array}{l}\text { 志 } \\
\text { की }\end{array}$} & Variable & Mean & Std Dev & t Value & $\operatorname{Pr}>|t|$ & $\mathbf{N}$ & Maximum & Minimum & Skewness & Kurtosis \\
\hline & $R_{i t}^{A D R}$ & 0.000254467 & 0.0189293 & 1.74 & 0.0824 & 16696 & 0.435351 & -0.21605 & 1.6543081 & 40.019418 \\
\hline & $R_{i t}^{\text {Equity }}$ & 0.00026279 & 0.0189447 & 1.79 & 0.0731 & 16696 & 0.230184 & -0.183033 & 0.2947042 & 12.8565739 \\
\hline & $R_{M t}^{\text {Home }}$ & 0.000453798 & 0.0174315 & 3.36 & 0.0008 & 16696 & 0.128812 & -0.129267 & -0.2066385 & 4.7712614 \\
\hline & $R_{M t}^{U S N Y S E}$ & 0.000234777 & 0.0124713 & 2.43 & 0.015 & 16696 & 0.115307 & -0.089683 & -0.0189981 & 8.1180301 \\
\hline & $R_{S t}^{F X}$ & 0.000158446 & 0.0112454 & -1.82 & 0.0687 & 16696 & 0.136331 & -0.146872 & -0.4131996 & 15.8421322 \\
\hline & Variable & Mean & Std Dev & t Value & $\operatorname{Pr}>|t|$ & $\mathbf{N}$ & Maximum & Minimum & Skewness & Kurtosis \\
\hline \multirow{5}{*}{$\begin{array}{l}\text { चे } \\
\frac{0}{0} \\
\overrightarrow{3} \\
n\end{array}$} & $R_{i t}^{A D R}$ & 0.000405647 & 0.0283433 & 1.31 & 0.191 & 8348 & 1 & -0.415195 & 4.8194553 & 198.9008524 \\
\hline & $R_{i t}^{\text {Equity }}$ & 0.000403614 & 0.026351 & 1.4 & 0.1617 & 8348 & 0.217797 & -0.249981 & -0.0273068 & 11.4641027 \\
\hline & $R_{M t}^{\text {Home }}$ & 0.000284552 & 0.0154548 & 1.68 & 0.0926 & 8348 & 0.141743 & -0.090888 & 0.1901074 & 5.7022643 \\
\hline & $R_{M t}^{U S N Y S E}$ & 0.000234777 & 0.0124717 & 1.72 & 0.0855 & 8348 & 0.115307 & -0.089683 & -0.0189998 & 8.1208216 \\
\hline & $R_{S t}^{F X}$ & 0.000092396 & 0.0045276 & 1.86 & 0.0623 & 8348 & 0.023856 & -0.025014 & 0.1223496 & 3.9833445 \\
\hline \multirow{6}{*}{ 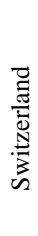 } & Variable & Mean & Std Dev & t Value & $\operatorname{Pr}>|t|$ & $\mathbf{N}$ & Maximum & Minimum & Skewness & Kurtosis \\
\hline & $R_{i t}^{A D R}$ & 0.000413987 & 0.0230653 & 2.32 & 0.0204 & 16696 & 1 & -0.616972 & 4.2230455 & 299.612307 \\
\hline & $R_{i t}^{\text {Equity }}$ & 0.000393737 & 0.0201827 & 2.52 & 0.0117 & 16696 & 0.234523 & -0.395833 & -0.4100334 & 27.7182984 \\
\hline & $R_{M t}^{\text {Home }}$ & 0.000381134 & 0.0185208 & 2.66 & 0.0078 & 16696 & 0.142846 & -0.097544 & 0.1949402 & 4.6779355 \\
\hline & $R_{M t}^{U S N Y S E}$ & 0.000234777 & 0.0124713 & 2.43 & 0.015 & 16696 & 0.115307 & -0.089683 & -0.0189981 & 8.1180301 \\
\hline & $R_{S t}^{F X}$ & 0.000029081 & 0.0079127 & 0.47 & 0.6349 & 16696 & 0.057974 & -0.041474 & 0.0898692 & 2.8538431 \\
\hline \multirow{6}{*}{ 荬 } & Variable & Mean & Std Dev & t Value & $\operatorname{Pr}>|t|$ & $\mathbf{N}$ & Maximum & Minimum & Skewness & Kurtosis \\
\hline & $R_{i t}^{A D R}$ & 0.000181684 & 0.0232677 & 1.33 & 0.182 & 29218 & 0.391304 & -0.381825 & 0.5910285 & 18.6116945 \\
\hline & $R_{i t}^{\text {Equity }}$ & 0.000265113 & 0.0255141 & 1.78 & 0.0757 & 29218 & 0.464262 & -0.381825 & 0.9962 & 18.9387828 \\
\hline & $R_{M t}^{\text {Home }}$ & 0.000198458 & 0.0104185 & 3.26 & 0.0011 & 29218 & 0.103254 & -0.069919 & -0.0177625 & 7.5265813 \\
\hline & $R_{M t}^{U S N Y S E}$ & 0.000234777 & 0.0124711 & 3.22 & 0.0013 & 29218 & 0.115307 & -0.089683 & -0.0189974 & 8.1168342 \\
\hline & $R_{S t}^{F X}$ & 0.000134521 & 0.007506 & 3.06 & 0.0022 & 29218 & 0.186981 & -0.086888 & 3.4540075 & 97.0535903 \\
\hline \multirow{6}{*}{ 离 } & Variable & Mean & Std Dev & t Value & $\operatorname{Pr}>|t|$ & $\mathbf{N}$ & Maximum & Minimum & Skewness & Kurtosis \\
\hline & $R_{i t}^{A D R}$ & 0.000495087 & 0.031512 & 1.02 & 0.3101 & 4174 & 0.507891 & -0.293062 & 1.3456482 & 29.1066938 \\
\hline & $R_{i t}^{\text {Equity }}$ & 0.000260471 & 0.0293843 & 0.57 & 0.5669 & 4174 & 0.369306 & -0.419506 & -0.024271 & 23.1834021 \\
\hline & $R_{M t}^{\text {Home }}$ & 0.00016593 & 0.0153671 & 0.7 & 0.4855 & 4174 & 0.085124 & -0.10346 & 0.0164624 & 2.9819084 \\
\hline & $R_{M t}^{U S N Y S E}$ & 0.000234777 & 0.0124724 & 1.22 & 0.224 & 4174 & 0.115307 & -0.089683 & -0.0190032 & 8.1264083 \\
\hline & $R_{S t}^{F X}$ & $-6.13 \mathrm{E}-06$ & 0.0030927 & -0.13 & 0.8981 & 4174 & 0.035612 & -0.034387 & 0.2473879 & 24.5913852 \\
\hline \multirow{6}{*}{ 光 } & Variable & Mean & Std Dev & t Value & $\operatorname{Pr}>|t|$ & $\mathbf{N}$ & Maximum & Minimum & Skewness & Kurtosis \\
\hline & $R_{i t}^{A D R}$ & 0.000309586 & 0.0242344 & 5.02 & $<.0001$ & 154438 & 1 & -0.780952 & 0.7127597 & 114.6890727 \\
\hline & $R_{i t}^{\text {Equity }}$ & 0.000511512 & 0.0306871 & 6.55 & $<.0001$ & 154438 & 1 & -0.780952 & 8.4434748 & 351.659368 \\
\hline & $R_{M t}^{\text {Home }}$ & 1.0870698 & 9.2913278 & 45.98 & $<.0001$ & 154438 & 99 & -0.999977 & 9.3811375 & 90.353229 \\
\hline & $R_{M t}^{U S N Y S E}$ & 0.000234777 & 0.0124709 & 7.4 & $<.0001$ & 154438 & 0.115307 & -0.089683 & -0.0189966 & 8.1155415 \\
\hline & $R_{S t}^{F X}$ & -0.000338354 & 0.0112985 & -11.77 & $<.0001$ & 154438 & 0.10568 & -0.214969 & -2.7571196 & 50.8026234 \\
\hline
\end{tabular}

\subsection{The Firm-Specific Empirical Time Series Models}

This research utilizes an adapted longitudinal Market Model for each of the ADR and equity shares which are specifically designed to capture the abnormal returns associated with (1) the earnings release date, and (2) the SEC Form 20-F filing date. More precisely, we employ qualitative variables to capture the impact of the earnings release date and the SEC Form 20-F file date upon the relation between the ADR share and equity share returns and their respective market-wide movements (i.e., the market average return). That is, we include earnings released and Form 20-F filing date qualitative variables (i.e., taking values of one on trading days surrounding the earnings release and Form 20-F filing dates and are zero valued all other trading days) to measure the abnormal returns associated with the earnings release and Form 20-F filing dates. Furthermore, we include the U.S. dollar reported earnings and U.S. GAAP earnings in the respective ADR and equity share return time series 
models in order to quantitatively measure the degree of association between the magnitudes reported and U.S. GAAP earnings and the respective abnormal equity and ADR share returns. The degree of association between earnings measures and abnormal security returns is taken as a measure of earnings quality and provides a vehicle for comparing the information conveyed at the earnings release with the information disclosed with the SEC Form 20-F filing.

We are particularly interested in the coefficients $\beta_{1 i}^{\text {Equity }}, \beta_{3 i}^{\text {Equity }}$, and $\beta_{1 i}^{A D R}, \beta_{3 i}^{A D R}$, which will measure the earning release date and SEC Form 20-F filing date abnormal returns in the ADR and home country equity share markets The earnings coefficients $\beta_{2 i}^{\text {Equity }}, \beta_{4 i}^{\text {Equity }}$, and $\beta_{2 i}^{A D R}, \beta_{4 i}^{A D R}$, will measure the degree of association between the magnitudes of earnings and magnitudes of abnormal returns associated with the earnings release and SEC Form 20-F filing date, providing a way of measuring the perceived quality of the disclosures as well as making comparisons.

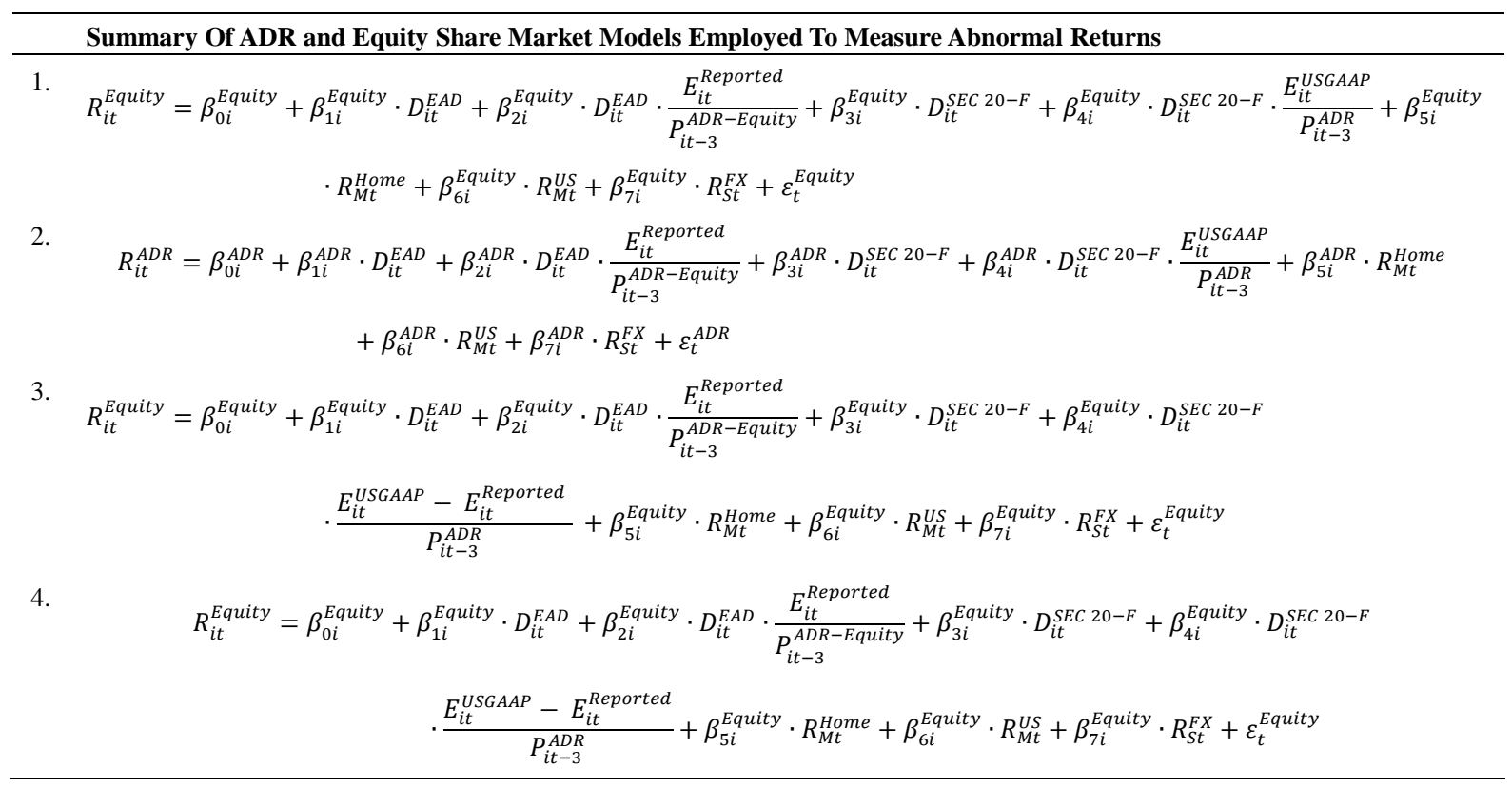

\section{Cross Section Statistics and Hypotheses Tests}

Having four measures of abnormal returns and four measures of associations between magnitudes of earning and magnitudes of abnormal returns produced by the firm-specific longitudinal models, we perform cross-sectional tests in order to investigate the statistical significance of the of the coefficients, as well as make statistical comparisons of their magnitudes. We first test whether the abnormal returns in the ADR and equity share markets that coincided with the earnings release date are statistically different from zero at the $\alpha=0.05$ confidence level (i.e., $\left.\mathrm{H}_{01}^{j k}: \beta_{1 i}^{k}=0 ; \mathrm{H}_{\mathrm{A} 1}^{j k}: \beta_{1 i}^{k} \neq 0, \forall \mathrm{k}: \mathrm{k}=1, \ldots, 4\right)$. Statistically significant abnormal ADR and equity share returns around the earnings release provides an indication that the earnings release conveys information to investors which they find useful in establishing ADR and equity share prices. Consequently, we interpret statistically significant abnormal returns as evidence substantiating the usefulness of reported earnings. Second, we test for the presence of abnormal ADR and equity share returns associated with the SEC Form 20-F filing date (i.e., $\left.\mathrm{H}_{02}^{j k}: \beta_{3 i}^{k}=0 ; \mathrm{H}_{\mathrm{A} 2}^{j k}: \beta_{3 i}^{k} \neq 0, \forall \mathrm{k}: \mathrm{k}=1, \ldots, 4\right)$. Statistically significant, abnormal ADR, and equity share returns around the SEC Form 20-F filing date provides an indication that the SEC disclosures communicate information to investors which they find useful in setting ADR and equity share prices. As a result, we interpret statistically significant (at the $\alpha=0.05$ confidence level) abnormal returns as evidence regarding the usefulness of SEC Form 20-F disclosures. Third, we perform statistical tests addressing the significance of the association between the magnitudes of reported earnings and the magnitudes of the abnormal ADR and equity share returns around the earnings release date ( i.e. $\mathrm{H}_{03}^{j k}: \beta_{2 i}^{k}=0 ; \mathrm{H}_{\mathrm{A} 3}^{j k}: \beta_{2 i}^{k} \neq 0, \forall \mathrm{k}: \mathrm{k}=1, \ldots, 4$ ). The presence of a statistically significant at the $\alpha=0.05$ confidence level association between magnitudes of reported earnings and magnitudes of ADR and equity share abnormal returns coinciding with the earnings release date is interpreted as evidence regarding the quality of reported earnings. Fourth, we perform statistical tests regarding the significance of the association between the magnitudes of U.S. GAAP earnings and the magnitudes of the 
abnormal ADR and equity share returns coinciding with the SEC Form 20-F filing date (i.e. $\mathrm{H}_{04}^{j k}: \beta_{4 i}^{k}=0 ; \mathrm{H}_{\mathrm{A4}}^{j k}$ : $\left.\beta_{4 i}^{k} \neq 0, \forall \mathrm{k}: \mathrm{k}=1, \ldots, 4\right)$. The statistical significance of the empirical association between magnitudes of U.S. GAAP earnings and the magnitudes of the abnormal ADR and equity share returns coinciding with the SEC Form 20-F filing date is interpreted as evidence regarding the quality of U.S. GAAP earnings. Next, we perform in the statistical comparison of the magnitudes of the ADR and equity share abnormal returns associated with the (earlier) earnings release date and the (later) SEC Form 20-F filing date (i.e., $\mathrm{H}_{05}^{j k}: \beta_{1 i}^{k}=\beta_{3 i}^{k} ; \mathrm{H}_{\mathrm{As}}^{j k}: \beta_{1 i}^{k} \neq \beta_{3 i}^{k}$, $\forall \mathrm{k}: \mathrm{k}=1, \ldots, 4)$. A statistically significant difference between the magnitudes of the two-disclosure date abnormal returns provides an indication regarding investors' perceived comparative usefulness of the two disclosures in evaluating ADR and equity share values. And last, we undertake a statistical comparison of the relative strength of the association between magnitudes of reported earnings and ADR and equity share abnormal returns associated with the earnings report date, and magnitudes of U.S. GAAP earnings and magnitudes of ADR and equity share abnormal returns associated with the SEC Form 20-F filing date (i.e., $\mathrm{H}_{06}^{j k}: \beta_{2 i}^{k}=\beta_{4 i}^{k} ; \mathrm{H}_{\mathrm{A6}}^{j k}: \beta_{2 i}^{k} \neq \beta_{4 i}^{k}$, $\forall \mathrm{k}: \mathrm{k}=1, \ldots, 4)$ ). A statistically significant (at the $\alpha=0.05$ confidence level) difference between the magnitudes of the two earnings-abnormal returns correlation measures yields evidence regarding investors' evaluation of comparative earnings quality for purposes of establishing ADR and equity share prices.

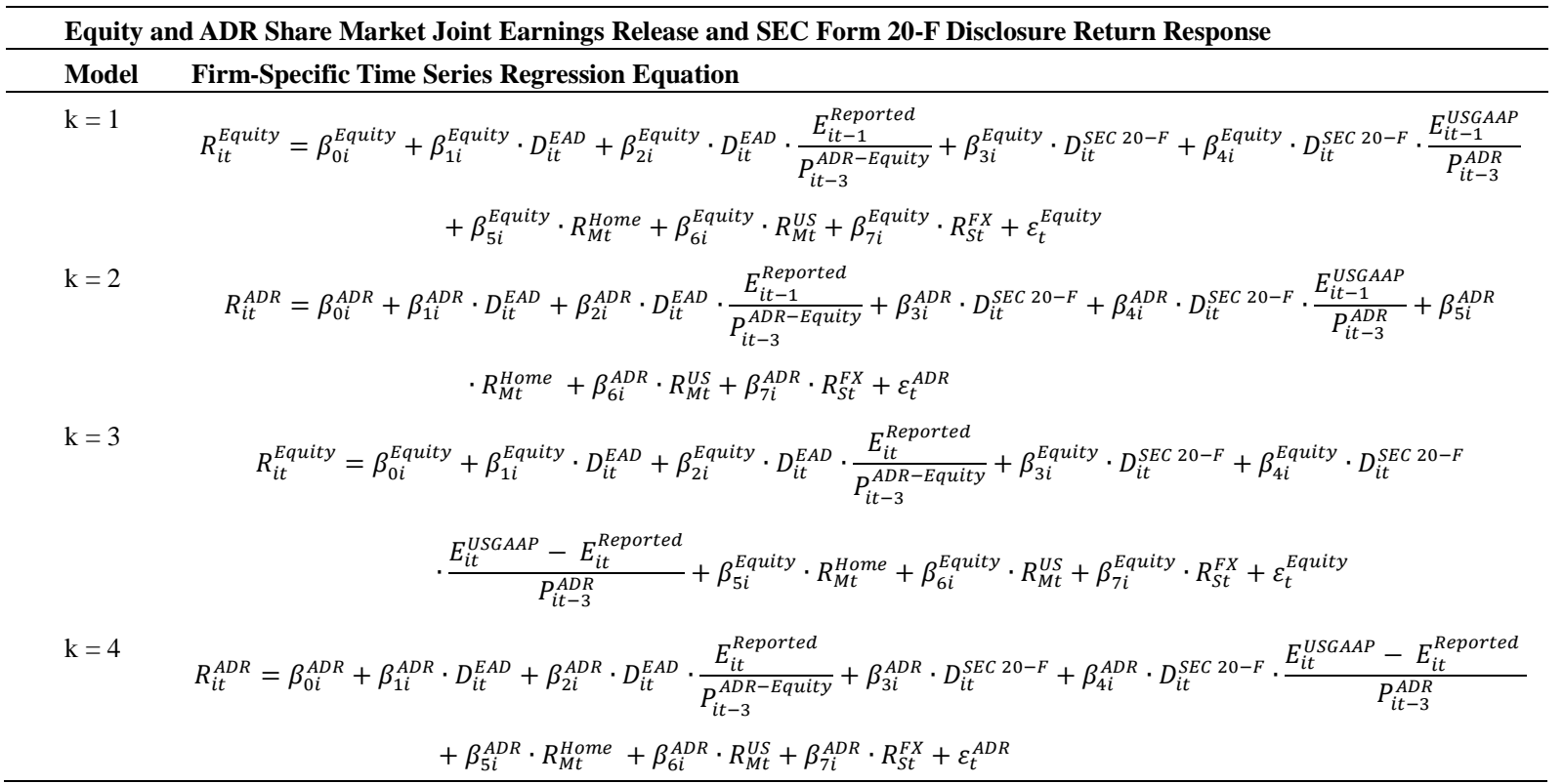

$\mathrm{H}_{01}^{j k}: \beta_{1 i}^{k}=0$

$\mathrm{H}_{\mathrm{A} 1}^{j k}: \beta_{1 i}^{k} \neq 0$

$\mathrm{H}_{03}^{j k}: \beta_{2 i}^{k}=0$

$\mathrm{H}_{\mathrm{A} 3}^{j k}: \beta_{2 i}^{k}>0$

$\mathrm{H}_{04}^{j k}: \beta_{4 i}^{k}=0$

$\mathrm{H}_{\mathrm{A} 4}^{j k}: \beta_{4 i}^{k}>0$

$\mathrm{H}_{05}^{j k}: \beta_{1 i}^{k}=\beta_{3 i}^{k}$

$\mathrm{H}_{\mathrm{A} 5}^{j k}: \beta_{1 i}^{k} \neq \beta_{3 i}^{k}$
We test the null hypothesis that the reported earnings release date indicator variable coefficient $\beta_{1 i}^{k}$ is equal to zero at the $\alpha=0.05$ confidence level. The alternative hypothesis is that the coefficient for the reported earnings release date indicator variable is significantly different from zero at the $\alpha=0.05$ confidence level. We conjecture that the $\mathrm{ADR}$ and equity share markets will display significant abnormal returns on the earnings release date.

We test the null hypothesis that the release date reported earnings magnitudes variable coefficient $\beta_{2 i}^{k}$ is equal to zero at the $\alpha=0.05$ confidence level. The alternative hypothesis is that the coefficient for the release date reported earnings magnitudes variable is significantly greater than zero at the $\alpha=0.05$ confidence level. We conjecture that the ADR and equity share release date abnormal returns will be proportional with magnitudes of reported earnings on the earnings report date.

We test the null hypothesis that the SEC Form 20-F filing date U.S. GAAP earnings magnitudes variable coefficient $\beta_{4 i}^{k}$ is equal to zero. The alternative hypothesis is that the coefficient for the SEC Form 20-F filing date U.S. GAAP earnings magnitudes variable is significantly greater than zero at the $\alpha=0.05$ confidence level. We conjecture that ADR and equity share markets will display significant abnormal returns which are proportional with SEC Form 20-F filing date U.S. GAAP earnings magnitudes.

We test the null hypothesis that the reported earnings release date and the SEC Form 20-F filing date ADR and equity market abnormal returns are equal to one another i.e., $\beta_{1 i}^{k}-\beta_{3 i}^{k}=0$. The alternative hypothesis is that the reported earnings release date and the SEC Form 20-F filing date ADR and equity market abnormal returns are significantly different from one another at the $\alpha=0.05$ confidence level. We conjecture that the relation between magnitudes of reported earnings release date and the SEC Form 20-F filing date ADR and equity market abnormal returns provides insight into the investors perceived quality of the comparative earnings disclosures. 


\begin{tabular}{ll}
\hline $\mathrm{H}_{06}^{j k}: \beta_{2 i}^{k}=\beta_{4 i}^{k}$ & We test the null hypothesis that the association of magnitudes of reported earnings and SEC Form 20-F U.S. GAAP \\
$\mathrm{H}_{\mathrm{A} 6}^{j k}: \beta_{2 i}^{k} \neq \beta_{4 i}^{k}$ & $\begin{array}{l}\text { earnings with earnings release date and SEC Form 20-F filing date abnormal returns are equal to one another i.e., } \\
\\
\end{array} \quad \beta_{2 i}^{k}-\beta_{4 i}^{k}=0$. The alternative hypothesis is that the association of magnitudes of reported earnings and SEC Form \\
& 20-F U.S. GAAP earnings with earnings release date and SEC Form 20-F filing date abnormal returns are \\
& coefficient significantly differ from one another zero at the $\alpha=0.05$ confidence level. We conjecture that differences \\
& in the association of magnitudes of release date earnings and the SEC Form 20-F filing date U.S. GAAP earnings \\
& with magnitudes of ADR and equity market abnormal returns provides insight into the investors' perceptions of the \\
& comparative quality of the earnings disclosures.
\end{tabular}

\section{Statistical Model Estimation and Results of Hypothesis Tests}

The results of the cross-sectional statistical tests addressing the significance and the quality of the time-series coefficients are shown in Table 2 through Table 5. It is apparent that, in general, virtually all of the time-series coefficient estimates are significantly different from zero. The first hypotheses that we test address whether the abnormal returns in the ADR and equity share markets that coincided with the earnings release date are statistically different from zero at the $\alpha=0.05$ confidence level ( i.e. $\left.\mathrm{H}_{01}^{j k}: \beta_{1 i}^{k}=0 ; \mathrm{H}_{\mathrm{A} 1}^{j k}: \beta_{1 i}^{k} \neq 0, \forall \mathrm{k}: \mathrm{k}=1, \ldots, 4\right)$. The results indicate that these null hypotheses are rejected at the $\alpha=0.05$ confidence level. Consequently, we conclude that statistically significant abnormal ADR and equity share returns occur around the earnings release dates. We interpret this result as a compelling indication that the earnings release conveys information to investors which they find useful in establishing ADR and equity share prices, and consider the finding substantiating evidence regarding the usefulness of reported earnings.

Table 2. Results of single equation ADR share market joint earnings release and sec form 20-F disclosure response

\begin{tabular}{|c|c|c|c|c|c|c|c|c|c|c|c|}
\hline \multicolumn{12}{|c|}{$\begin{array}{l}\text { Mean Coefficient Values for Equity Return Equation No.1: The equity return equations were estimated of } 354 \text { firms using a time series of daily } \\
\text { home country equity returns over the } 16 \text {-year period from } 2000 \text { to } 2015 \text {. For each firm time series Ordinary Least Squares estimation techniques were } \\
\text { employed to estimate the coefficients. Descriptive Statistics for the coefficient values and related hypotheses tests are shown below. }\end{array}$} \\
\hline Model Variable & \multicolumn{4}{|c|}{ Descriptive Statistics } & \multicolumn{3}{|c|}{ Related Hypotheses Tests } & \multicolumn{4}{|c|}{ Hypotheses Tests Results } \\
\hline \multirow[t]{5}{*}{$\beta_{0 i}^{\text {Equity }}$ Intercept } & \multicolumn{4}{|c|}{ Basic Statistical Measures } & \multicolumn{7}{|c|}{ Tests for Location: $\mathrm{H}_{0:} \beta_{0 i}^{\text {Equity }}=0$} \\
\hline & \multicolumn{2}{|l|}{ Location } & \multicolumn{2}{|l|}{ Variability } & \multicolumn{3}{|l|}{ Test Statistic } & \multicolumn{4}{|l|}{ p Value } \\
\hline & Mean & 0.000302 & Std Dev & 0.000335 & Student's t & $\mathrm{t}$ & 23.9709 & $\operatorname{Pr}>|t|$ & $<.0001$ & \$ & Reject \\
\hline & Median & 0.000298 & Variance & 0.000000 & Sign & M & 263.0000 & $\operatorname{Pr}>=|M|$ & $<.0001$ & + & Reject \\
\hline & Mode & 0.000295 & Range & 0.006080 & Signed Rank & S & 110851.5000 & $\operatorname{Pr}>=|S|$ & $<.0001$ & * & Reject \\
\hline$\beta_{1 i}^{\text {Equity }}$ Earning & \multicolumn{4}{|c|}{ Basic Statistical Measures } & \multicolumn{7}{|c|}{ Tests for Location: $\beta_{1 i}^{\text {Equity }}=0$} \\
\hline \multirow[t]{4}{*}{ Release Date } & \multicolumn{2}{|l|}{ Location } & \multicolumn{2}{|l|}{ Variability } & Test & \multicolumn{2}{|c|}{ Statistic } & \multicolumn{4}{|l|}{ p Value } \\
\hline & Mean & 0.002940 & Std Dev & 0.007120 & Student's t & $\mathrm{t}$ & 10.9810 & $\operatorname{Pr}>|t|$ & $<.0001$ & + & Reject \\
\hline & Median & 0.004460 & Variance & 0.000051 & Sign & M & 166.0000 & $\operatorname{Pr}>=|M|$ & $<.0001$ & \$ & Reject \\
\hline & Mode & -0.002560 & Range & 0.063990 & Signed Rank & $S$ & 68082.0000 & $\operatorname{Pr}>=|S|$ & $<.0001$ & * & Reject \\
\hline$\beta_{2 i}^{\text {Equity }}$ Reported & \multicolumn{4}{|c|}{ Basic Statistical Measures } & \multicolumn{7}{|c|}{ Tests for Location: $\beta_{2 i}^{\text {Equity }}=0$} \\
\hline \multirow[t]{4}{*}{ Earnings } & \multicolumn{2}{|l|}{ Location } & \multicolumn{2}{|l|}{ Variability } & Test & \multicolumn{2}{|c|}{ Statistic } & \multicolumn{4}{|l|}{$\mathrm{p}$ Value } \\
\hline & Mean & 0.007995 & Std Dev & 0.131670 & Student's t & $\mathrm{t}$ & 1.5845 & $\operatorname{Pr}>|t|$ & 0.1135 & & \\
\hline & Median & 0.002068 & Variance & 0.017340 & Sign & M & 168.0000 & $\operatorname{Pr}>=|M|$ & $<.0001$ & * & Reject \\
\hline & Mode & 0.000000 & Range & 3.844640 & Signed Rank & S & 59818.0000 & $\operatorname{Pr}>=|S|$ & $<.0001$ & * & Reject \\
\hline$\beta_{3 i}^{\text {Equity }}$ SEC Form 20-F & \multicolumn{4}{|c|}{ Basic Statistical Measures } & \multicolumn{7}{|c|}{ Tests for Location: $\beta_{3 i}^{\text {Equity }}=0$} \\
\hline Filing Date & Location & & Variability & & Test & Stat & & $\mathrm{p}$ Value & & & \\
\hline & Mean & 0.002681 & Std Dev & 0.006230 & Student's t & $\mathrm{t}$ & 11.2102 & $\operatorname{Pr}>|t|$ & $<.0001$ & & Reject \\
\hline & Median & 0.003160 & Variance & 0.000039 & Sign & M & 160.0000 & $\operatorname{Pr}>=|\mathrm{M}|$ & $<.0001$ & + & Reject \\
\hline & Mode & 0.000000 & Range & 0.110790 & Signed Rank & S & 66752.0000 & $\operatorname{Pr}>=|S|$ & $<.0001$ & * & Reject \\
\hline$\beta_{4 i}^{\text {Equity }}$ U.S. GAAP & Basic Stat & stical Measu & & & Tests for & & $\beta_{4 \mathrm{i}}^{\text {Equity }}=0$ & & & & \\
\hline Earnings & Location & & Variability & & Test & Stat & & $\mathrm{p}$ Value & & & \\
\hline & Mean & 0.004654 & Std Dev & 0.014040 & Student's t & $\mathrm{t}$ & 8.2952 & $\operatorname{Pr}>|t|$ & $<.0001$ & & Reject \\
\hline & Median & 0.001610 & Variance & 0.000197 & Sign & M & 147.5000 & $\operatorname{Pr}>=|\mathrm{M}|$ & $<.0001$ & \$ & Reject \\
\hline & Mode & 0.000000 & Range & 0.235290 & Signed Rank & S & 45941.0000 & $\operatorname{Pr}>=|S|$ & $<.0001$ & * & Reject \\
\hline$\beta_{5 i}^{\text {Equity }}$ Equity Market & Basic Sta & stical Measu & ures & & Tests for Loc & & & & & & \\
\hline Return & Location & & Variability & & Test & & & $\mathrm{p}$ Value & & & \\
\hline & Mean & 0.064672 & Std Dev & 0.077170 & Student's t & $\mathrm{t}$ & 21.9015 & $\operatorname{Pr}>|t|$ & $<.0001$ & & Reject \\
\hline & Median & 0.033796 & Variance & 0.005960 & Sign & M & 281.0000 & $\operatorname{Pr}>=|M|$ & $<.0001$ & * & Reject \\
\hline & Mode & 0.000000 & Range & 0.589400 & Signed Rank & $\mathrm{S}$ & 102901.0000 & $\operatorname{Pr}>=|S|$ & $<.0001$ & * & Reject \\
\hline
\end{tabular}




\begin{tabular}{|c|c|c|c|c|c|c|c|c|c|c|c|}
\hline \multirow{3}{*}{$\begin{array}{l}\beta_{6 i}^{\text {Equity }} \text { U.S. ADR Market } \\
\text { Return }\end{array}$} & \multicolumn{4}{|c|}{ Basic Statistical Measures } & \multicolumn{7}{|c|}{ Tests for Location: $\beta_{6 i}^{\text {Equity }}=0$} \\
\hline & \multicolumn{2}{|l|}{ Location } & \multicolumn{2}{|l|}{ Variability } & \multirow{2}{*}{$\begin{array}{l}\text { Test } \\
\text { Student's t }\end{array}$} & \multicolumn{2}{|c|}{ Statistic } & \multicolumn{4}{|l|}{$\mathrm{p}$ Value } \\
\hline & Mean & 0.315411 & Std Dev & 0.353820 & & $\mathrm{t}$ & 23.7200 & $\operatorname{Pr}>|t|$ & $<.0001$ & $*$ & Reject \\
\hline & Median & 0.064900 & Variance & 0.125190 & Sign & $\mathrm{M}$ & 352.0000 & $\operatorname{Pr}>=|M|$ & $<.0001$ & \$ & Reject \\
\hline & Mode & 0.022471 & Range & 1.416730 & Signed Rank & $\mathrm{S}$ & 125125.0000 & $\operatorname{Pr}>=|S|$ & $<.0001$ & + & Reject \\
\hline$\beta_{7 i}^{\text {Equity }}$ Home Country & \multicolumn{4}{|c|}{ Basic Statistical Measures } & \multicolumn{7}{|c|}{ Tests for Location: $\beta_{7 i}^{\text {Equity }}=0$} \\
\hline \multirow[t]{4}{*}{ Currency Spot Rate } & Location & & Variability & & Test & & & $\mathrm{p}$ Value & & & \\
\hline & Mean & 0.078292 & Std Dev & 0.183310 & Student's t & $\mathrm{t}$ & 10.9724 & $\operatorname{Pr}>|t|$ & $<.0001$ & $*$ & Reject \\
\hline & Median & 0.055776 & Variance & 0.033600 & Sign & M & 210.0000 & $\operatorname{Pr}>=|\mathrm{M}|$ & $<.0001$ & + & Reject \\
\hline & Mode & 0.000000 & Range & 3.249340 & Signed Rank & $\mathrm{S}$ & 69429.5000 & $\operatorname{Pr}>=|S|$ & $<.0001$ & $*$ & Reject \\
\hline
\end{tabular}

\begin{tabular}{lllll}
\hline Hypothesis Test: $\mathrm{H}_{0}: \beta_{1 i}^{\text {Equity }}=\beta_{3 i}^{\text {Equity }}$ & $\operatorname{Pr}>=|\mathbf{S}|$ & $\mathbf{0 . 0 0 1 8} *$ & Reject & Hypothesis Test: $\mathrm{H}_{0}: \beta_{2 i}^{\text {Equity }}=\beta_{4 i}^{\text {Equity }} \operatorname{Pr}>=|\mathbf{S}| \mathbf{0 . 3 8 6 2}$ Not Reject \\
\hline
\end{tabular}

1: Tests the null hypothesis that the abnormal returns associated with the earnings release and the SEC Form 20-F filing date in ADR share market are equal to one another. Table 2 indicates that the null hypothesis of no difference is rejected at the $\alpha=0.05$ confidence level. Consequently, we conclude that the abnormal returns associated with the earnings release are larger than the abnormal returns associated with the SEC Form 20-F filing date in the ADR share market.

2: Tests the null hypothesis that there is no difference in the association between magnitudes of earnings and magnitudes of abnormal returns at the earnings release and the SEC Form 20-F filing date in the ADR share market. Table 2 indicates that the null hypothesis of no difference is rejected at the $\alpha=0.05$ confidence level. Consequently, we conclude that magnitudes of U.S. GAAP earnings are more highly associated with abnormal returns in the ADR share market at the SEC Form 20-F disclosure date than are similar magnitudes of reported earnings at the earnings release date. This result suggests that U.S. GAAP Earnings are perceived as being higher quality than home country earnings in the ADR share market.

Table 3. Results of single equation ADR share market joint earnings release and SEC form 20-F disclosure response

Mean Coefficient Values for ADR Return Equation No.2: The U.S. listed ADR return equations were estimated of 354 firms using a time series of daily home country equity returns over the 16-year period from 2000 to 2015. For each firm time series Ordinary Least Squares estimation techniques were employed to estimate the coefficients. Descriptive Statistics for the coefficient values and related hypotheses tests are shown below.

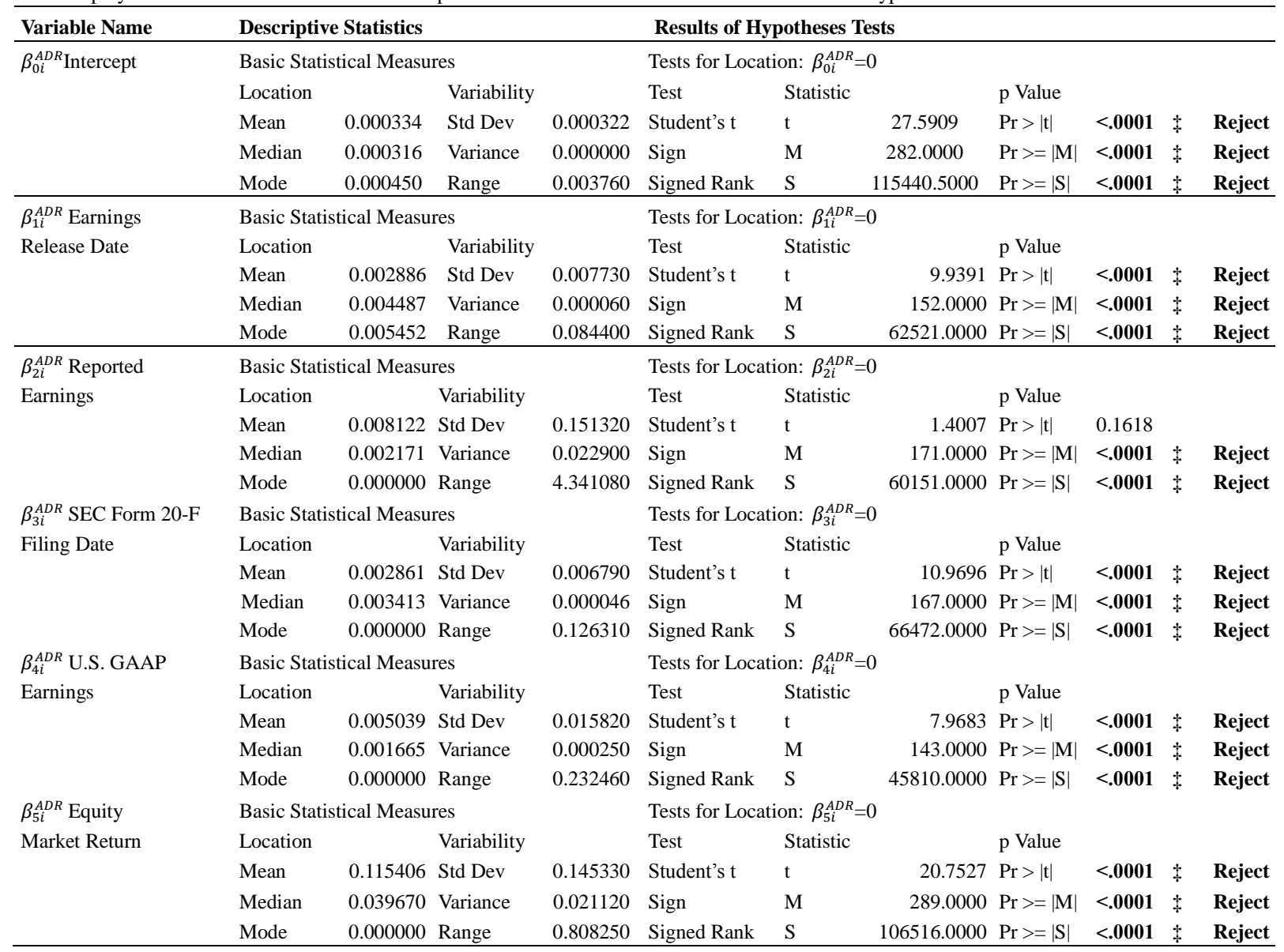




\begin{tabular}{|c|c|c|c|c|c|c|c|c|c|c|c|}
\hline$\beta_{6 i}^{A D R} \mathrm{ADR}$ & \multicolumn{4}{|c|}{ Basic Statistical Measures } & \multicolumn{7}{|c|}{ Tests for Location: $\beta_{6 i}^{A D R}=0$} \\
\hline \multirow{4}{*}{ Market Return } & \multicolumn{2}{|l|}{ Location } & \multicolumn{2}{|l|}{ Variability } & \multirow{2}{*}{$\begin{array}{l}\text { Test } \\
\text { Student's t }\end{array}$} & \multicolumn{2}{|l|}{ Statistic } & \multicolumn{4}{|l|}{$\mathrm{p}$ Value } \\
\hline & Mean & 0.151604 & Std Dev & 0.201870 & & $\mathrm{t}$ & 19.9830 & $\operatorname{Pr}>|t|$ & $<.0001$ & $\$$ & Reject \\
\hline & Median & 0.039000 & Variance & 0.040750 & Sign & M & 310.0000 & $\operatorname{Pr}>=|M|$ & $<.0001$ & + & Reject \\
\hline & Mode & 0.037107 & Range & 1.179110 & Signed Rank & $\mathrm{S}$ & 115700.0000 & $\operatorname{Pr}>=|S|$ & $<.0001$ & $*$ & Reject \\
\hline$\beta_{7 i}^{A D R}$ Daily & \multicolumn{4}{|c|}{ Basic Statistical Measures } & \multicolumn{7}{|c|}{ Tests for Location: $\beta_{7 i}^{A D R}=0$} \\
\hline \multirow[t]{4}{*}{ Exchange Rate } & \multicolumn{2}{|l|}{ Location } & \multicolumn{2}{|l|}{ Variability } & Test & Statistic & & $\mathrm{p}$ Value & & & \\
\hline & Mean & 0.083998 & Std Dev & 0.152950 & Student's t & $\mathrm{t}$ & 14.1091 & $\operatorname{Pr}>|t|$ & $<.0001$ & 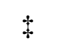 & Reject \\
\hline & Median & 0.059607 & Variance & 0.023390 & Sign & M & 217.0000 & $\operatorname{Pr}>=|M|$ & $<.0001$ & + & Reject \\
\hline & Mode & 0.000000 & Range & 1.732340 & Signed Rank & $\mathrm{S}$ & 72073.0000 & $\operatorname{Pr}>=|S|$ & $<.0001$ & + & Reject \\
\hline Hypothesis Tes & $i^{D R}=\beta_{3 i}^{A D R}$ & $\operatorname{Pr}>=|S|$ & $0.0018 *$ & Reject & Hypothesis T & st: $\mathrm{H}_{0}: \beta_{2 i}^{A D R}$ & $=\beta_{4 i}^{A D R}$ & $\operatorname{Pr}>=|S| \quad 0$ & $0.3862 \mathrm{~N}$ & NOT & Reject \\
\hline
\end{tabular}

1: Tests the null hypothesis that the abnormal returns associated with the earnings release and the SEC Form 20-F filing date in the ADR market are equal to one another. Table No. 3 indicates that the null hypothesis of no difference is rejected at the $\alpha=0.05$ confidence level. Consequently, we conclude that the abnormal returns associated with the earnings release are larger than the abnormal returns associated with the SEC Form 20-F filing date in the ADR share market.

2: Tests the null hypothesis that there is no difference in the association between magnitudes of earnings and magnitudes of abnormal returns at the earnings release and the SEC Form 20-F filing date in the ADR share market. Table No.3 indicates that the null hypothesis of no difference is rejected at the $\alpha=0.05$ confidence level. Consequently, we conclude that magnitudes of U.S. GAAP earnings are more highly associated with abnormal returns in the ADR share market at the SEC Form 20-F disclosure date than are similar magnitudes of reported earnings at the earnings release date. This result suggests that U.S. GAAP Earnings are perceived as being higher quality than home country earnings in the ADR share market.

Table 4. Results of single equation ADR share return joint earnings release and SEC form 20-F disclosure response

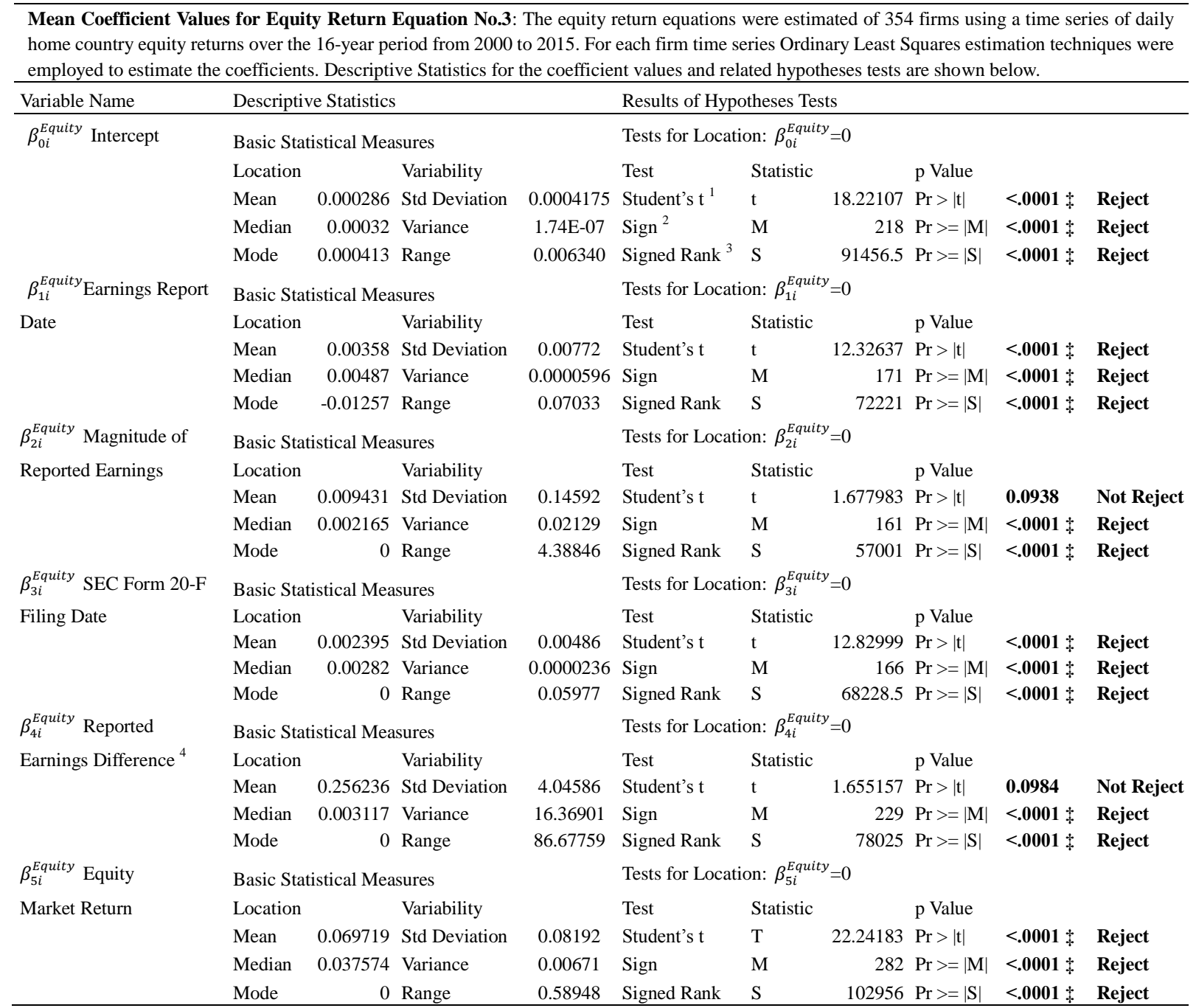




\begin{tabular}{|c|c|c|c|c|c|c|c|c|c|c|}
\hline$\beta_{6 i}^{\text {Equity }} \mathrm{ADR}$ & \multicolumn{4}{|c|}{ Basic Statistical Measures } & \multicolumn{6}{|c|}{ Tests for Location: $\beta_{6 i}^{\text {Equity }}=0$} \\
\hline \multirow[t]{4}{*}{ Market Return } & \multicolumn{2}{|c|}{ Location } & \multicolumn{2}{|l|}{ Variability } & \multicolumn{3}{|c|}{ Test $\quad$ Statistic } & \multicolumn{3}{|l|}{$\mathrm{p}$ Value } \\
\hline & Mean & 0.360536 & Std Deviation & 0.39452 & Student's t & $\mathrm{T}$ & 24.31634 & $\operatorname{Pr}>|t|$ & $<.0001+$ & Reject \\
\hline & Median & 0.07533 & Variance & 0.15564 & Sign & M & 352 & $\operatorname{Pr}>=|\mathrm{M}|$ & $<.0001+$ & Reject \\
\hline & Mode & 0.028389 & Range & 1.84525 & Signed Rank & S & 125132 & $\operatorname{Pr}>=|S|$ & $<.0001+$ & Reject \\
\hline$\beta_{7 i}^{\text {Equity }}$ Daily Spot & \multicolumn{4}{|c|}{ Basic Statistical Measures } & \multicolumn{6}{|c|}{ Tests for Location: $\beta_{7 i}^{\text {Equity }}=0$} \\
\hline \multirow[t]{4}{*}{ Exchange Rate Change } & \multicolumn{2}{|l|}{ Location } & \multicolumn{2}{|l|}{ Variability } & Test & \multicolumn{2}{|c|}{ Statistic } & \multicolumn{3}{|l|}{$\mathrm{p}$ Value } \\
\hline & Mean & 0.085636 & Std Deviation & 0.2048 & Student's t & $\mathrm{T}$ & 10.74238 & $\operatorname{Pr}>|t|$ & $<.0001 \ddagger$ & Reject \\
\hline & Median & 0.064255 & Variance & 0.04194 & Sign & M & 199 & $\operatorname{Pr}>=|\mathrm{M}|$ & $<.0001 \ddagger$ & Reject \\
\hline & Mode & 0 & Range & 3.24523 & Signed Rank & $S$ & 62505 & $\operatorname{Pr}>=|S|$ & $<.0001+$ & Reject \\
\hline
\end{tabular}

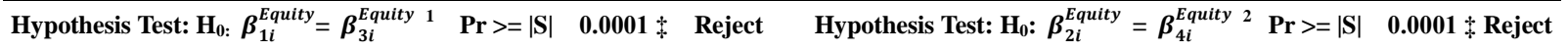

1: Tests the null hypothesis that the abnormal returns associated with the earnings release and the SEC Form 20-F filing date in the home country equity market are equal to one another. Table No.4 indicates that the null hypothesis of no difference is rejected at the $\alpha=0.05$ confidence level. Consequently, we conclude that the abnormal returns associated with the earnings release are larger than the abnormal returns associated with the SEC Form 20-F filing date in the home country equity market.

2: Tests the null hypothesis that there is no difference in the association between magnitudes of earnings and magnitudes of abnormal returns at the earnings release and the SEC Form 20-F filing date in the home country equity market. Table No.4 indicates that the null hypothesis of no difference is rejected at the $\alpha=0.05$ confidence level. Consequently, we conclude that magnitudes of U.S. GAAP earnings are more highly associated with abnormal returns in the home country equity market at the SEC Form 20-F disclosure date than are similar magnitudes of reported earnings at the earnings release date. This result suggests that U.S. GAAP Earnings are perceived as being higher quality than home country earnings in the home country equity market.

Table 5. Results of single equation ADR share market joint earnings release and SEC form 20-F disclosure response

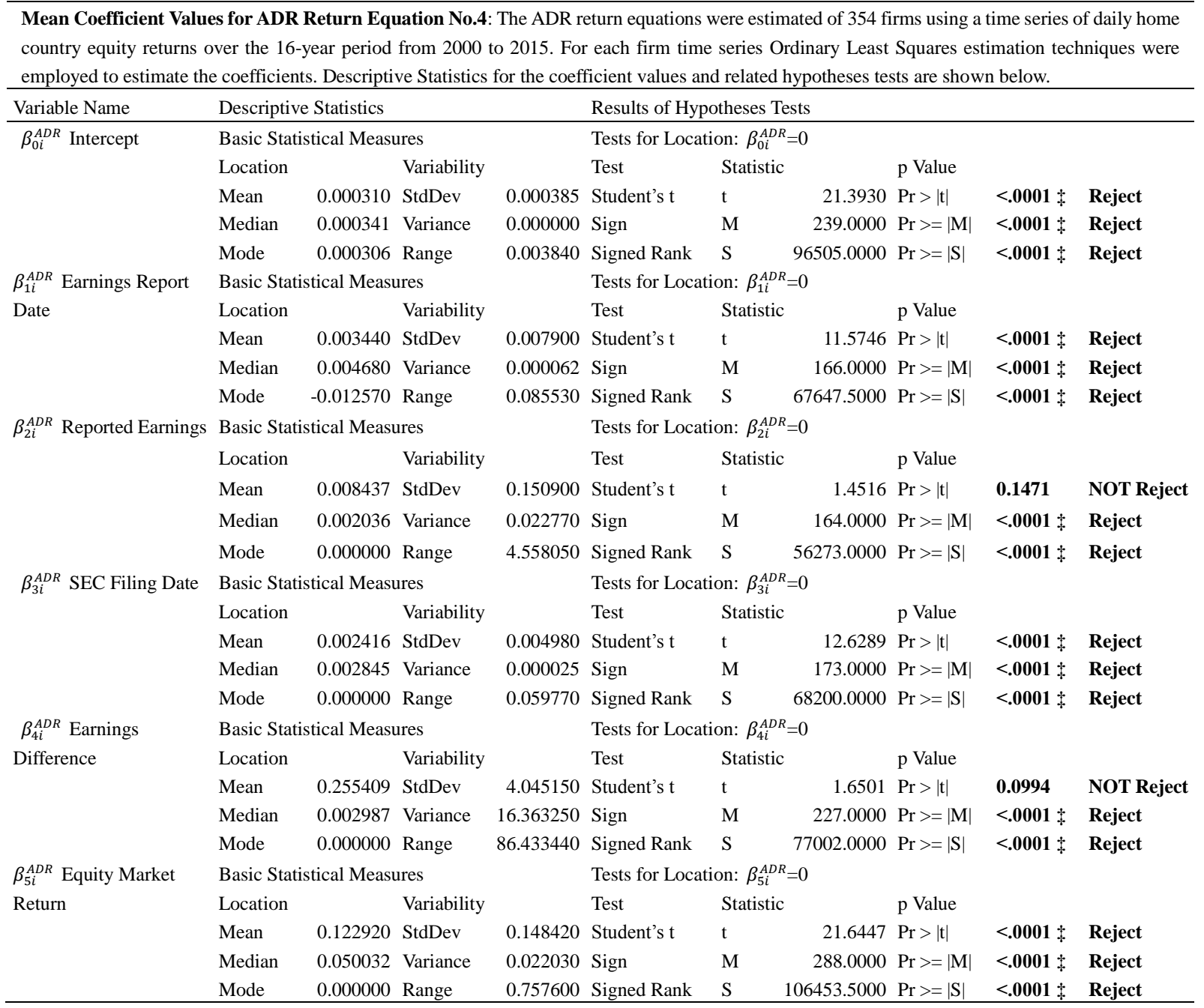




\begin{tabular}{|c|c|c|c|c|c|c|c|c|c|c|}
\hline$\beta_{6 i}^{A D R}$ ADR Market & \multicolumn{4}{|c|}{ Basic Statistical Measures } & \multicolumn{6}{|c|}{ Tests for Location: $\beta_{6 i}^{A D R}=0$} \\
\hline \multirow[t]{4}{*}{ Return } & \multicolumn{2}{|l|}{ Location } & \multicolumn{2}{|l|}{ Variability } & \multirow{2}{*}{$\begin{array}{l}\text { Test } \\
\text { Student's t }\end{array}$} & \multicolumn{2}{|c|}{ Statistic } & \multicolumn{3}{|l|}{$\mathrm{p}$ Value } \\
\hline & Mean & 0.176220 & StdDev & 0.244260 & & $\mathrm{t}$ & 19.1967 & $\operatorname{Pr}>|t|$ & $<.0001 \ddagger$ & Reject \\
\hline & Median & 0.049024 & Variance & 0.059660 & Sign & M & 309.0000 & $\operatorname{Pr}>=|\mathrm{M}|$ & $<.0001 \ddagger$ & Reject \\
\hline & Mode & 0.022251 & Range & 1.478300 & Signed Rank & $\mathrm{S}$ & 115295.0000 & $\operatorname{Pr}>=|S|$ & $<.0001 \dagger$ & Reject \\
\hline \multirow{5}{*}{$\begin{array}{c}\beta_{7 i}^{A D R} \text { Daily Spot } \\
\text { Exchange Rate }\end{array}$} & \multicolumn{4}{|c|}{ Basic Statistical Measures } & \multicolumn{6}{|c|}{ Tests for Location: $\beta_{7 i}^{A D R}=0$} \\
\hline & Location & & Variability & & Test & Sta & & $\mathrm{p}$ Value & & \\
\hline & Mean & 0.094277 & StdDev & 0.182790 & Student's t & $\mathrm{t}$ & 13.2501 & $\operatorname{Pr}>|t|$ & $<.0001 \ddagger$ & Reject \\
\hline & Median & 0.068137 & Variance & 0.033410 & Sign & M & 212.0000 & $\operatorname{Pr}>=|\mathrm{M}|$ & $<.0001 \ddagger$ & Reject \\
\hline & Mode & 0.000000 & Range & 1.903640 & Signed Rank & $\mathrm{S}$ & 66882.0000 & $\operatorname{Pr}>=|S|$ & $<.0001 \ddagger$ & Reject \\
\hline
\end{tabular}

\begin{tabular}{llllllll}
\hline Hypothesis Test: $\mathrm{H}_{0}: \beta_{1 i}^{A D R}=\beta_{3 i}^{A D R}$ & $\operatorname{Pr}>=|\mathrm{S}|$ & $\mathbf{0 . 0 0 0 1} \neq$ & Reject & Hypothesis Test: $\mathrm{H}_{0}: \boldsymbol{\beta}_{2 i}^{A D R}=\boldsymbol{\beta}_{4 i}^{A D R}$ & $\operatorname{Pr}>=|\mathrm{S}|$ & $0.0001 \neq$ & Reject
\end{tabular}

1: Tests the null hypothesis that the abnormal returns associated with the earnings release and the SEC Form 20-F filing date in ADR share market are equal to one another. Table No.5 indicates that the null hypothesis of no difference is rejected at the $\alpha=0.05$ confidence level. Consequently, we conclude that the abnormal returns associated with the earnings release are larger than the abnormal returns associated with the SEC Form 20-F filing date in the ADR share market.

2: Tests the null hypothesis that there is no difference in the association between magnitudes of earnings and magnitudes of abnormal returns at the earnings release and the SEC Form 20-F filing date in the ADR share market. Table No.5 indicates that the null hypothesis of no difference is rejected at the $\alpha=0.05$ confidence level. Consequently, we conclude that magnitudes of U.S. GAAP earnings are more highly associated with abnormal returns in the ADR share market at the SEC Form 20-F disclosure date than are similar magnitudes of reported earnings at the earnings release date. This result suggests that U.S. GAAP Earnings are perceived as being higher quality than home country earnings in the ADR share market.

The second set of hypotheses which we test investigate the presence of abnormal ADR and equity share returns associated with the SEC Form 20-F filing date ( i.e. $\mathrm{H}_{02}^{j k}: \beta_{3 i}^{k}=0 ; \mathrm{H}_{\mathrm{A2}}^{j k}: \beta_{3 i}^{k} \neq 0, \forall \mathrm{k}: \mathrm{k}=1, \ldots, 4$ ). This set of null hypotheses is rejected are rejected at the $\alpha=0.05$ confidence level. Consequently, we conclude that statistically significant abnormal ADR and equity share returns are observed coinciding with the SEC Form 20-F filing date providing an indication that the SEC disclosures communicate information to investors which they find useful in setting ADR and equity share prices. Furthermore, we interpret results as providing convincing evidence regarding the usefulness of SEC Form 20-F disclosures.

The third set of hypotheses regard the significance of the association between the magnitudes of reported earnings and the magnitudes of the abnormal ADR and equity share returns around the earnings release date (i.e., $\left.\mathrm{H}_{03}^{j k}: \beta_{2 i}^{k}=0 ; \mathrm{H}_{\mathrm{A} 3}^{j k}: \beta_{2 i}^{k} \neq 0, \forall \mathrm{k}: \mathrm{k}=1, \ldots, 4\right)$. The hypothesis that the coefficient is equal to zero in cross-section is rejected at the $\alpha=0.05$ confidence level. We interpret the presence of a statistically significant at the $\alpha=0.05$ confidence level association between magnitudes of reported earnings and magnitudes of ADR and equity share abnormal returns coinciding with the earnings release date as providing reliable evidence regarding the quality of reported earnings.

The fourth set of hypotheses concerns the significance of the association between the magnitudes of U.S. GAAP earnings and the magnitudes of the abnormal ADR and equity share returns coinciding with the SEC Form 20-F filing date (i.e., $\left.\mathrm{H}_{04}^{j k}: \beta_{4 i}^{k}=0 ; \mathrm{H}_{\mathrm{A} 4}^{j k}: \beta_{4 i}^{k} \neq 0, \forall \mathrm{k}: \mathrm{k}=1, \ldots, 4\right)$. Once again, the hypothesis that the coefficient is equal to zero in cross-section is rejected at the $\alpha=0.05$ confidence level. The statistical significance of the empirical association between magnitudes of U.S. GAAP earnings and the magnitudes of the abnormal ADR and equity share returns coinciding with the SEC Form 20-F filing date is interpreted as evidence regarding the quality of U.S. GAAP earnings.

Next, we perform the statistical comparison of the magnitudes of the ADR and equity share abnormal returns associated with the (earlier) earnings release date and the (later) SEC Form 20-F filing date (i.e., $\mathrm{H}_{05}^{j k}: \beta_{1 i}^{k}=\beta_{3 i}^{k}$; $\left.\mathrm{H}_{\mathrm{A} 5}^{j k}: \beta_{1 i}^{k} \neq \beta_{3 i}^{k}, \forall \mathrm{k}: \mathrm{k}=1, \ldots, 4\right)$. The null hypothesis concerning the equality of abnormal returns at the two dates in cross-section is rejected at the $\alpha=0.05$ confidence level. We observe marginally larger abnormal returns at the earnings release date than that the SEC form 20 as the date and the statistically significant difference between the magnitudes of the two-disclosure date abnormal returns provides an indication regarding investors' perceived comparative usefulness of the two disclosures in evaluating ADR and equity share values.

Finally, we undertake a statistical comparison of the relative strength of the association between magnitudes of reported earnings and ADR and equity share abnormal returns associated with the earnings report date, and magnitudes of U.S. GAAP earnings and magnitudes of ADR and equity share abnormal returns associated with the SEC Form 20-F filing date (i.e., $\left.\mathrm{H}_{06}^{j k}: \beta_{2 i}^{k}=\beta_{4 i}^{k} ; \mathrm{H}_{\mathrm{A} 6}^{j k}: \beta_{2 i}^{k} \neq \beta_{4 i}^{k}, \forall \mathrm{k}: \mathrm{k}=1, \ldots, 4\right)$ ). The hypothesis concerning the equality of the earnings coefficients in cross-section at the two dates is rejected at the $\alpha=0.05$ confidence 
level. Consequently, we conclude that there is a statistically significant (at the $\alpha=0.05$ confidence level) difference between the magnitudes of the two earnings-abnormal returns correlation measures and that U.S. GAAP earnings exhibit a higher degree of association with ADR and equity share returns than reported earnings. These results provide compelling evidence regarding investors' evaluation of comparative earnings quality for purposes of establishing ADR and equity share prices.

\section{Conclusions and Suggestions for Future Research}

We investigate the information content of non-U.S. firms' earnings releases vis-à-vis the SEC Form 20-F filings in both ADR and home country equity share markets. We employ models of the ADR and equity security share earnings release date abnormal returns controlling for the incremental firm-specific SEC Form 20-F disclosures required of exchange-listed ADR's. Our results suggest that both ADR and home country equity share markets exhibit abnormal returns associated with the earnings release date and the SEC Form 20-F filing date with the earnings report date abnormal returns marginally larger. Particularly noteworthy, however, is the association between magnitudes of U.S. GAAP earnings and magnitudes of SEC Form 20-F filing date abnormal returns is significantly larger than the association between magnitudes of reported earnings and earnings report date abnormal returns in both the ADR and home country equity share markets. Our results seem to suggest a perception that the U.S. GAAP rules are of higher quality and may provide an indication that the U.S. ADR share market response dominates the cross-market information flow, driving the home country equity share market response in a manner consistent with the notion that U.S. GAAP conveys price relevant information beyond reported earnings for non-U.S. firms. We conjecture that non-U.S. firms choosing to be listed on the major U.S. exchanges will comply with the supplemental disclosure requirements in order to have the supplemental disclosures impounded in the home country equity share price via the ADR share price in the manner described by Fishman and Hagerty (1989).

\section{References}

Agarwal, S., Liu, C., \& Rhee, S. G. (2007). Where does price discovery occur for stocks traded in multiple markets? Evidence from Hong Kong and London. Journal of International Money and Finance, 26, 46-63. https://doi.org/10.1016/j.jimonfin.2006.10.011

Amir, E., Harris, T., \& Venuti, E. (1993). A comparison of the value-relevance of U.S. vs. non-U.S. GAAP accounting measures using Form 20-F reconciliations. Journal of Accounting Research, 31, 230-264. https://doi.org/10.2307/2491172

Ashbaugh, H. (2001). Non-U.S. firms' accounting standard choices. Journal of Accounting and Public Policy, 20 (Summer), 129-153. https://doi.org/10.1016/S0278-4254(01)00025-4

Ashbaugh, H., \& Pincus, M. (2001). Domestic accounting standards, International accounting standards, and the predictability of earnings. Journal of Accounting Research, 39(December), 417-434. https://doi.org/10.1111/1475-679X.00020

Bailey, W., Karolyi, G. A., \& Salva, C. (2006). The Economic Consequences of Increased Disclosure: Evidence from International Cross-Listings. Journal of Financial Economics, 80, 175-213. https://doi.org/10.1016/j.jfineco.2005.06.002

Baker, H. K., Nofsinger, J., \& Weaver, D. (2002). International Cross-Listing and Visibility. Journal of Financial and Quantitative Analysis, 37, 495-521. https://doi.org/10.2307/3594990

Ball, R., Kothari, S. P., \& Robin, A. (2000). The effect of international institutional factors on properties of accounting earnings. Journal of Accounting and Economics, 29, 1-51. https://doi.org/10.1016/S0165-4101(00)00012-4

Ball, R., Kothari, S. P., \& Wu, J. S. (2003). Incentives versus standards: Properties of accounting earnings in four East Asian countries. Journal of Accounting and Economics, 36, 235-270. https://doi.org/10.1016/j.jacceco.2003.10.003

Barth, M., Landsman, W., \& Lang, M. H. (2008). International accounting standards andaccountingquality. Journal of Accounting Research, 46(June), 467-498. https://doi.org/10.1111/j.1475-679X.2008.00287.x

Barth, M., Landsman, W., Lang, M. H., \& Williams, C. (2006). Accounting quality: International accounting standards and U.S. GAAP. Working paper, University of North Carolina.

Bartov, E., Goldberg, S., \& Kim, M. (2005). Comparative value relevance among German, U.S., and international accounting standards: A German stock market perspective. Journal of Accounting Auditing 
and Finance, 20, 95-119. https://doi.org/10.1177/0148558X0502000201

Binder, J. J. (1998). The Event Study Methodology since 1969. Review of Quantitative Finance and Accounting, 11, 111-137. https://doi.org/10.1023/A:1008295500105

Bradshaw, M., Callahan, C., Ciesielski, J., ... \& Yohn, T. (2010). Roadmap for the potential use of financial statements prepared in accordance with International Financial Reporting Standards (IFRS) by US issuers. Accounting Horizons, 24, 117-28. https://doi.org/10.2308/acch.2010.24.1.117

Bris, A., Cantale, S., \& Nishiotis, G. (2007). A Breakdown of the Valuation Effects of International Cross-Listing. European Financial Management, 13(3), 298-530. https://doi.org/10.1111/j.1468-036X.2006.00370.x

Chamberlain, G. (1982). Multivariate Regression Models for Panel Data. Journal of Econometrics, 18, 5-46. https://doi.org/10.1016/0304-4076(82)90094-X

Chan, K., \& Hameed, A. (2006). Stock Price Synchronicity and Analyst Coverage in Emerging Markets. Journal of Financial Economics, 80, 115-147. https://doi.org/10.1016/j.jfineco.2005.03.010

Chemmanur, T., \& Paolo, F. (2006). Competition and Co-operation among Exchanges: A Theory of Cross Listing and Endogenous Listing Standards. Journal of Financial Economics, 82, 455-489. https://doi.org/10.1016/j.jfineco.2005.06.008

Chen, C. J. P., Gul, F. A., \& Su, X. J. (1999). A comparison of reported earnings under Chinese GAAP vs. IAS: evidence from the Shanghai Stock Exchange. Accounting Horizons, 13(2), 91-111. https://doi.org/10.2308/acch.1999.13.2.91

Chen, L., \& Sami, H. (2008). Trading volume reaction to the earnings reconciliation from IAS to U.S. GAAP. Contemporary Accounting Research, 25, 15-33. https://doi.org/10.1506/car.25.1.1

Chen, L., \& Sami, H. (2010). Trading volume reaction to the earnings reconciliation from IFRS to U.S. GAAP: Further evidence. Working paper, Arizona State University and Lehigh University.

Chen, L., \& Samim, H. (2010). The Impact of Firm Characteristics on Trading Volume Reaction to the Earnings Reconciliation from IFRS to U.S. GAAP. Contemporary Accounting Research, 30(2), 697-718. https://doi.org/10.1111/j.1911-3846.2012.01168.x

Christensen, H., Lee, E., \& Walker, M. (2009). Do IFRS reconciliations convey information? The effect of debt contracting. Journal of Accounting Research, 47, 1167-1248. https://doi.org/10.1111/j.1475-679X.2009.00345.x

Coffee, J. (2002). Racing Towards the Top? The Impact of Cross-Listings and Stock Market Competition on International Corporate Governance. Columbia Law Review, 102, 1757-1831. https://doi.org/10.2307/1123661

Dargenidou, C., Stuart, M., \& Ivana, R. (2006). Expected Earnings Growth and the Cost of Capital: An Analysis of Accounting Regime Change in the European Financial Market. Unpublished Working Paper, Paper Number 06/12. https://doi.org/10.1111/j.1467-6281.2006.00207.x

Daske, H. (2006). Economic benefits of adopting IFRS or US-GAAP: Have the expected costs of equity capital really decreased? Journal of Business Finance and Accounting, 33, 329-373. https://doi.org/10.1111/j.1468-5957.2006.00611.x

Daske, H., \& Gebhardt, G. (2006). International Financial Reporting Standards and experts' perceptions of disclosure quality. Abacus, 42, 461-498. https://doi.org/10.1111/j.1467-6281.2006.00211.x

Daske, H., Hail, L., Leuz, C., \& Verdi, R. (2008). Mandatory IFRS reporting around the world: Early evidence on the economic consequences. Journal of Accounting Research, 46, 1085-1142. https://doi.org/10.1111/j.1475-679x.2008.00306.x

Daske, H., Hail, L., Leuz, C., \& Verdi, R. (2009). Adopting a Label: Heterogeneity in the Economic Consequences of IFRS Adoptions. Working Paper.

Ding, D. K., Harris, F. H., Lau, S. T., \& McInish, T. H. (1999). An Investigation of Price Discovery in Informationally-Linked Markets: Equity Trading in Malaysia and Singapore. Journal of Multinational Financial Management, 9, 317-329. https://doi.org/10.1016/S1042-444X(99)00005-5

Doidge, C. (2005). What is the Effect of Cross-Listing on Corporate Ownership and Control? Unpublished working paper, University of Toronto.

Doidge, C., Karolyi, G. A., \& Stulz, R. (2004). Why are foreign firms listed in the U.S. worth more? Journal of 
Financial Economics, 71, 205-238. https://doi.org/10.1016/S0304-405X(03)00183-1

Domowitz, I., Glen, J., \& Madhavan, A. (1998). International Cross Listing and Order Flow Migration: Evidence from an Emerging Market. Journal of Finance, 53, 2001-2027. https://doi.org/10.1111/0022-1082.00081

Easley, D., O’Hara, M., \& Paperman, J. (1998). Financial Analysts and Information-Based Trade. Journal of Financial Markets, 1, 175-201. https://doi.org/10.1016/S1386-4181(98)00002-0

Errunza, V., \& Miller, D. (2000). Market Segmentation and the Cost of Capital in International Equity Markets. Journal of Financial and Quantitative Analysis, 35, 577-600. https://doi.org/10.2307/2676256

Eun, C. S., \& Sabherwal, S. (2003). Price Discovery for Internationally Traded Securities: Evidence from the U.S.-Listed Canadian Stocks. Journal of Finance, 58, 549-576. https://doi.org/10.1111/1540-6261.00537

Fernandes, N., \& Ferreira, M. (2008). Does International Cross-Listing Improve the Informational Environment. Journal of Financial Economics, 88, 216-244. https://doi.org/10.1016/j.jfineco.2007.06.002

Fishman, M. J., \& Kathleen, M. H. (1989). Disclosure Decisions by Firms and the Competition for Price Efficiency. The Journal of Finance, 44(3), 633-646. https://doi.org/10.1111/j.1540-6261.1989.tb04382.x

Foerster, S., \& Karolyi, G. A. (1999). The Effects of Market Segmentation and Investor Recognition on Asset Prices: Evidence from Foreign Stocks Listing in the US. Journal of Finance, 54, 981-1013. https://doi.org/10.1111/0022-1082.00134

Francis, J. R., Kurana, I. K., Martin, X., Pereira, R. (2008). The role of firm-specific incentives and country factors in explaining voluntary IAS adoptions: Evidence from private firms. European Accounting Review, 1, 1-30. https://doi.org/10.1080/09638180701819899

Gordon, E., Jorgensen, B., \& Linthicum, C. (2009). Could IFRS replace U.S. GAAP? A comparison of earnings attributes and informativeness in the U.S. market. Working paper, Temple University, Columbia University, and University of Texas - San Antonio.

Grammig, J., Melvin, M., \& Schlag, C. (2005). Internationally Cross-Listed Stock Prices During Overlapping Trading Hours: Price Discovery and Exchange Rate Effects. Journal of Empirical Finance, 12, 139-164. https://doi.org/10.1016/j.jempfin.2003.10.004

Hail, L., \& Leuz, C. (2004). Cost of Capital and Cash Flow Effects of US Cross-Listings. Unpublished working paper, European Corporate Governance Institute.

Hail, L., \& Leuz, C. (2009). Cost of capital effects and changes in growth expectations around U.S. cross-listings. Journal of Financial Economics, 93, 428-454. https://doi.org/10.1016/j.jfineco.2008.09.006

Harris, M., \& Muller, K. (1999). The market valuation of IAS versus U.S. GAAP accounting measures using form 20-F reconciliations. Journal of Accounting and Economics, 26, 285-312. https://doi.org/10.1016/S0165-4101(99)00003-8

Haverty, J. L. (2006). Are IFRS and U.S. GAAP converging? Some evidence from People's Republic of China companies listed on the New York Stock Exchange. Journal of International Accounting, Auditing and Taxation, 15, 48-71. https://doi.org/10.1016/j.intaccaudtax.2006.01.004

Healy, P., Hutton, A., \& Palepu, K. (1999). Stock Performance and Intermediation Changes Surrounding Sustained Increases in Disclosure. Contemporary Accounting Research, 16, 485-520. https://doi.org/10.1111/j.1911-3846.1999.tb00592.x

Henry, E., Lin, S., \& Yang, Y. (2009). The European-U.S. GAAP gap: amount, type, homogeneity and value relevance of IFRS to U.S. GAAP from 20-F reconciliations. Accounting Horizons, 23, 121-150. https://doi.org/10.2308/acch.2009.23.2.121

Hopkins, P. E., Botosan, C. A., Bradshaw, M. T., Callahan, C. M. et al. (2008). Response to the SEC Release. Acceptance from Foreign Private Issuers of Financial Statements Prepared in Accordance with International Financial Reporting Standards without Reconciliation to U.S. GAAP File No. S7-13-07. Accounting Horizons, June, 223-240. https://doi.org/10.2308/acch.2008.22.2.223

Hupperets, E. C. J., \& Menkveld, A. J. (2002). Intraday Analysis of Market Integration: Dutch Blue Chips traded in Amsterdam and New York. Journal of Financial Markets, 5, 57-82. https://doi.org/10.1016/S1386-4181(01)00019-2

Jamal, K. G., Benston, J., Carmichael, D. R., ... \& Watts, R. L. (2008). A perspective on the SEC's proposal to accept financial statements prepared in accordance with international financial reporting standards (IFRS) 
without reconciliation to U.S. GAAP. Accounting Horizons, June, 241-248. https://doi.org/10.2308/acch.2008.22.2.241

Jiang, J., Petroni, K. R., \& Wang, I. Y. (2010). Did eliminating the 20-F reconciliation between IFRS and US GAAP matter? Working paper. Michigan State University.

Kang, T., Krishnan, G., Wolfe, M., \& Yi, H. (2010). The Impact of Eliminating 20-F Reconciliation Requirement for IFRS Filers on Earnings Predictability and Information Quality. Working paper.

Karolyi, G. A. (2006). The World of Cross-Listings and Cross-Listings of the World: Challenging Conventional Wisdom. Review of Finance, 10, 73-115. https://doi.org/10.1007/s10679-006-6980-8

Kim, J. B., \& Shi, H. (2007). International financial reporting standards, institutional infrastructures, and cost of equity capital around the world. Working Paper, The Hong Kong Polytechnic University.

Kim, J. B., Tsui, J., \& Yi, C. H. (2007). The voluntary adoptions of international accounting standards and loan contracting around the world. Working Paper, The Hong Kong Polytechnic University.

Kim, M., Szakmary, A. C., \& Mathur, I. (2000). Price Transmission Dynamics between ADRs and Their Underlying Foreign Securities. Journal of Banking and Finance, 24, 1359-1382. https://doi.org/10.1016/S0378-4266(99)00076-X

Kim, O., \& Verrecchia, R. (2001). The relation among returns, disclosure and trading volume information. Accounting Review, 76, 633-654. https://doi.org/10.2308/accr.2001.76.4.633

Lang, M. H., Raedy, J., \& Yetman, M. (2006). How representative are firms that are cross-listed in the United States? An Analysis of Accounting Quality. Journal of Accounting Research, 41(2), 363-386. https://doi.org/10.1111/1475-679X.00108

Lang, M., \& Lundholm, R. (1996). Corporate Disclosure Policy and Analyst Behavior. Accounting Review, 71, 467-492.

Lang, M., Lins, K., \& Miller, D. (2003a). ADRs, Analysts, and Accuracy: Does Cross Listing in the US Improve a Firm's Information Environment and Increase Market Value? Journal of Accounting Research, 41, 317-346. https://doi.org/10.1111/1475-679X.00106

Lang, M., Raedy, J., \& Wilson, W. (2006). Earnings Quality and Cross Listing: Are Reconciled Earnings Comparable to US Earnings? Journal of Accounting and Economics, 42, 255-283. https://doi.org/10.1016/j.jacceco.2006.04.005

Lapointe-Antunes, P., Cormier, M. M., \& Gay-Angers, S. (2006). On the relationship between Voluntary Disclosure, Earnings Smoothing and the Value relevance of Earnings: The case of Switzerland. European Accounting Review, 15(4),.465-505. https://doi.org/10.1080/09638180601102040

Lau, S. T., \& Diltz, J. D. (1994). Stock Returns and the Transfer of Information between the New York and Tokyo Stock Exchanges. Journal of International Money and Finance, 13, 211-222. https://doi.org/10.1016/0261-5606(94)90016-7

Leuz, C. (2003). IAS versus U.S. GAAP: Information asymmetry-based evidence from Germany's new market. Journal of Accounting Research, 41, 445-472. https://doi.org/10.1111/j.1475-679X.2003.00111.x

Leuz, C. (2006). Cross listing, bonding and firms' reporting incentives: A discussion of Lang, Raedy and Wilson. Journal of Accounting and Economics, 42, 285-299. https://doi.org/10.1016/j.jacceco.2006.04.003

Leuz, C., \& Verrecchia, R. (2000). The economic consequences of increased disclosure. Journal of Accounting Research, 38, 91-124. https://doi.org/10.2307/2672910

Leuz, C., Nanda, D., \& Wysocki, P. (2003). Earnings Management and Investor Protection: An International $\begin{array}{lllll}\text { Comparison. Journal of Financial } & \text { Economics, }\end{array}$ https://doi.org/10.1016/S0304-405X(03)00121-1

Licht, A. (2003). Cross-Listing and Corporate Governance: Bonding or Avoiding? Chicago Journal of International Law, 4, 141-163. https://doi.org/10.2139/ssrn.382660

Lungarella, M., Ishiguro, K., Kuniyoshi, Y., \& Otsu, N. (in press). Methods for quantifying the causal structure of bivariate time series. International Journal of Bifurcation and Chaos. https://doi.org/10.1142/s0218127407017628

Pereda, E., Quiroga, R. Q., \& Bhattacharya, J. (2005). Nonlinear multivariate analysis of neurophysiological signals. ProgNeurobiol, 77, 1-37. https://doi.org/10.1016/j.pneurobio.2005.10.003 
Phylaktis, K., \& Korczak, P. (2004). Price Discovery Process in International Cross- Listings: Evidence from US-Listed British and French Companies. Working Paper, Cass Business School.

Plumlee, M., \& Plumlee, D. (2007). 20-F filers and SEC proposed changes: Some evidence of a U.S. home GAAP preference. Working paper, University of Utah.

Pownall, G., \& Schipper, K. (1999). Implications of accounting research for the SEC's consideration of International Accounting Standards for U.S. securities offerings. Accounting Horizons, 13, 259-280. https://doi.org/10.2308/acch.1999.13.3.259

Reese, W., \& Weisbach, M. (2002). Protection of minority shareholder interests, cross-listing in the United States, and subsequent equity offerings. Journal of Financial Economics, 66, 65-104. https://doi.org/10.1016/S0304-405X(02)00151-4

Roulstone, D. (2003). Analyst Following and Market Liquidity. Contemporary Accounting Research, 20, 1-27. https://doi.org/10.1506/X45Y-PMH7-PNYK-4ET1

Schreiber, T. (2000). Measuring information transfer. Phys. Rev. Lett., 85, 461-4. https://doi.org/10.1103/PhysRevLett.85.461

SEC Release No. 33-8818. (July 2, 2007). Proposed Rule: Acceptance From Foreign Private Issuers Of Financial Statements Prepared In Accordance With International Financial Reporting Standards Without Reconciliation To U.S. GAAP.

SEC Release No. 33-8879. (December 21, 2007). Final Rule: Acceptance From Foreign Private Issuers of Financial Statements Prepared In Accordance With International Financial Reporting Standards Without Reconciliation To U.S. GAAP. Retrieved from http://www.sec.gov/rules/final/2007/33-8879.pdf

Soderstrom, N. S., \& Sun, K. J. (2007). IFRS adoption and accounting quality: A review. European Accounting Review, 16, 675-702. https://doi.org/10.1080/09638180701706732

\section{Notes}

Note 1. Extant research has examined cross-market information linkages focusing upon the relation among ADR and security returns and trading volume. Eun and Shim (1989), Karolyi (1995), and Chen, Chiang, and So (2003) investigate the relation among larger market returns and King and Wadhwani (1990), Bae and Karolyi (1994), Kanas (1998), and Ng (2000) examine the relation among larger and emerging markets returns, concluding that the U.S. markets are dominant for returns in the sense that information flows from the U.S. market to other global markets. Furthermore, Lee and Rui (2002), Gagnon and Karolyi (2003), and Kim (2005) report research results indicating that U.S. markets are dominant for trading volume in the sense that information flows from the U.S. market to other global markets.

Note 2. Foreign private issuers submitting the periodic update Form 20-F accompanied by financial statements prepared in accordance with IFRS need not include the reconciliation and components to U.S. GAAP Net Income and Shareholders Equity amounts (i.e., Item 17 or Item 18 of Form 20-F) provided that (1) the foreign private issuer unequivocally and explicitly indicates that its financial statements are in compliance with English language IFRS in an appropriate footnote accompanying the financial statements, and (2) the independent auditor's report accompanying the foreign private issuer's financial statements affirms that those financial statements are presented in accordance with English language version IFRS. Foreign private issuers continue to include a U.S. GAAP reconciliation with the Form $20-\mathrm{F}$ in any one of the following circumstances: (1) the foreign private issuer's financial statements contain a departure from IASB IFRS; (2) the foreign private issuer does not indicate unreservedly and explicitly that the financial statements are presented in accordance with IASB IFRS; (3) the foreign private issuer's independent auditor does not opine on compliance with IASB IFRS; and(4) the auditor's report contains any qualification relating to compliance with IASB IFRS.

Note 3. The extant research literature, to date, however, conveys sparse direct evidence indicating that non U.S. firms share cross-listing contributes to the price formation processes in the home country equity shares. Employing variation of firm-specific returns as a proxy for the stock price informativeness construct, Fernando and Ferreira (2008) investigate the impact of the cross-listing decision upon home country equity share price informativeness and report a statistically significant positive relation between U.S cross-listing and home country equity share price informativeness. Bailey et al. (2006) examine the impact of U.S cross-listing upon the magnitude of price and volume reactions to (earnings announcements) contending that more private information equates with higher return volatility reasoning that diminishing return volatility following upon U.S cross-listing 
may indicate less disagreement among investors regarding interpretation of the earnings announcement. Their results indicate that both absolute return and absolute volume reactions to earnings announcements increase significantly following upon firms' cross-listing of shares on U.S. equity exchanges. Other studies in the extant research literature investigate the impact of non-U.S. firms share cross-listing upon analysts' forecasts and media coverage as surrogates for the firm's information environment. Specifically, Lang et al. (2003) and Baker et al. (2002) report that cross-listed firms derive greater analyst following as well as more accurate earnings forecasts.

Note 4. Naturally, the costs of firm disclosures may be incurred by both foreign private issuers as well as investors. Of course, the costs associated with firms producing and communicating disclosures, including auditing and legal costs, are nontrivial. However, there is also a nontrivial cost to investors of acquiring and assimilating the information. While firm costs associated with producing and disseminating information have been examined in the extant research literature, the costs associated with investors acquiring and assimilating information remains relatively unexamined. We conjecture that the different accounting principles employed by foreign private issuers in the preparation of their financial statements, in numerous instances, represents a nontrivial cost to U.S. investors which is mitigated to a large extent by the SEC Form 20-F reconciliation to U.S. GAAP.

Note 5. One stream of extant research literature suggests that more analyst coverage and more accurate earnings forecasts lead to an improved information environment (Lang \& Lundholm, 1996; Healy et al., 1999). Baker et al. (2002) report finding increased visibility, as measured by analyst and media coverage, around the time of cross-listing. Results reported by Lang et al. (2003a) suggest that non-U.S. firms listed on U.S. exchanges benefit from increased analyst coverage and more accurate forecasts. Bailey et al. (2006) report research results indicating greater volatility and trading activity around earnings announcements following upon cross-listing of developed market firms. Although a preponderance of the evidence indicates a positive association between the information environment and cross-listing, the relation remains ambiguous for the following reasons:

1) The increased disclosure requirement following upon U.S. exchange cross-listing may substitute for the collection of private information to the extent that a smaller amount of firm-specific information would be impounded into stock prices (Kim \& Verrecchia, 2001).

2) Easley et al. (1998) and Roulstone (2003) suggest that analyst activity is not necessarily a reliable indicator of private information trading since analysts themselves are more of a conduit and do not have significant firm-specific information. Moreover, Piotroski and Roulstone (2004) research results indicating that increased analyst coverage stimulates the production of industry and market-wide information and undermines firm-specific stock return variation. Chan and Hameed (2006) report research results indicating that greater analyst coverage results in decreased firm-specific return variation in emerging markets.

3) The cross-listing information environment effect may vary across countries. The improved disclosure following upon U.S. ADR cross listing cross-listing may have a differential impact on the home country environment. Ball (2001) suggests that improving accounting disclosures by itself is insufficient to substantially improve information environment. A diverse range of other country specific economic, legal, and political infrastructure modifications are needed in order to improve the actual quality of financial reporting. Licht (2003) and Siegel (2005) assert that the voluntary disclosure following upon U.S. cross-listing permits firms to bond their reputations to U.S. disclosure requirements. Lang et al. (2006) report that the added regulation by the SEC remains ineffective, but rather that U.S. listed ADRs home environment remains important in explaining the quality of its U.S. GAAP-reported earnings.

Note 6. Financial reporting environment quality is determined, in part, by firms' selection of accounting standards but also by socio-economic institutional degree of implementation of auditing and enforcement incentives (e.g., Ball, 2001; Ball et al., 2003). Consequently, although IFRS are considered sufficiently high quality standards, firms' financial statements may be unreliable due to poorly implemented auditing and enforcement incentives among foreign private issuers' home countries.

Note 7. Since U.S. GAAP is generally perceived by investors as constituting the standard for "high-quality" accounting standards, the SEC Form 20-F reconciling differences with U.S. GAAP earnings and equity impose important constraints on management accounting policy choices. The effective SEC Form 20-F management accounting policy choice constraint arises as a result of the need to minimize the reconciling differences with U.S. GAAP in communicating to investors the relative success of their prospective investment projects in order for investors to perceive the ADR as maintaining similarly "high-quality" reporting practices as other firms at their comparative market stature. Quite naturally, the more pronounced the differences with U.S. GAAP earnings and equity raise important question regarding earnings management practices. Furthermore, Luez (2006) 
conjectures that more pronounced the differences with U.S. GAAP may also have the effect of motivating local authorities to convey additional scrutiny. In any event, the more pronounced differences with U.S. GAAP may garner increased investor uncertainty in relation to the parameters of the underlying earnings process (Chen \& Sami, 2008; Leuz, 2006). Results reported by Bradshaw (2004) suggest that levels of U.S. ownership of non-U.S. firms is greater for ADRs reporting smaller SEC Form 20-F reconciling U.S. GAAP differences. Likewise, Plumlee and Plumlee (2007) report that ADRs reporting smaller SEC Form 20-F reconciling U.S. GAAP differences experienced comparatively higher levels of trading.

Note 8. Early research such as Harris and Mueller (1999) provides statistically significant research results indicating that U.S. GAAP earnings convey security price relevant information beyond that communicated with IFRS. Employing a sample of 31 firms over the years 1992-1996 (i.e., a total of 89 firm year observations), they regress U.S. GAAP earnings and earnings changes expressed in terms of the decomposed IFRS and home country earnings and earnings changes and their respective Form 20-F reconciliation amounts and changes in the respective Form 20-F reconciliation amounts onto annual equity securities returns. More specifically, they find that the IFRS to U.S. GAAP reconciliation amount is statistically significant in its association with annual equity securities returns at conventional confidence levels (i.e., p. 302 Table 4 Panel B). Harris and Mueller employ a security returns v. earnings levels model identical to the one used by Amir et al. (1993) and Barth and Clinch (1996) to investigate the security price relevance of Home Country GAAP vs. U.S. GAAP differences and corroborate their findings that the SEC Form $20-F$ reconciliation amounts are significantly associated with equity security returns. The Amir et al. (1993) research examines Form 20-F reconciliations over the years 1981-1991 and utilizes a sample of 101 firms.

\section{Copyrights}

Copyright for this article is retained by the author(s), with first publication rights granted to the journal.

This is an open-access article distributed under the terms and conditions of the Creative Commons Attribution license (http://creativecommons.org/licenses/by/4.0/). 